\title{
Proton Structure Functions from Chiral Dynamics and QCD Constraints
}

\author{
H. J. Weber \\ Institute of Nuclear and Particle Physics, University of Virginia, \\ Charlottesville, VA 22903, USA
}

\begin{abstract}
The spin fractions and deep inelastic lepton structure functions of the proton are analyzed using chiral field theory involving Goldstone bosons. A detailed comparison with recent chiral models sheds light on their successful description of the spin fractions of the proton as being due to neglecting helicity non-flip chiral transitions. This approximation is valid for zero mass but not for constituent quarks. Since the chiral spin fraction models with the pure spin-flip approximation reproduce the measured spin fractions of the proton, axialvector constituent-quark-Goldstone boson couplings are found to be inconsistent with the proton spin data. Initial quark valence distributions are then constructed using quark counting constraints at Bjorken $x \rightarrow 1$ and Regge behavior at $x \rightarrow 0$. Sea quark distributions predicted by chiral field theory on this basis have correct order of magnitude and shape. The spin fractions also agree with the data.
\end{abstract}

PACS numbers: 11.30.Rd, 12.39.Fe, 14.20.Dh

Keywords: Deep inelastic scattering, chiral field theory, QCD constraints 


\section{INTRODUCTION}

The nonrelativistic quark model (NQM) explains qualitatively many of the strong, electromagnetic and weak properties of the nucleon and other octet (and decuplet) baryons in terms of three valence quarks whose dynamics is motivated by quantum chromodynamics (QCD), the gauge field theory of the strong interaction. The effective degrees of freedom at the scale $\Lambda_{Q C D}$ are dressed or dynamical quarks along with Goldstone bosons which are expected to emerge in the spontaneous chiral symmetry breakdown $(\chi S B)$ of QCD that is characterized by quark condensates. While the NQM description of baryon states has not been derived from first principles of QCD, solutions of Schwinger-Dyson equations for light quarks with models for color confinement lead to a momentum dependent quark mass $m_{q}\left(p^{2}\right)$. Such dynamical quarks become constituent quarks when $m_{q}\left(p^{2}\right)$ is approximated by a constant $m_{q}(0)$ at the scale $\Lambda_{Q C D}$. In Nambu-Jona-Lasinio models [1] of the $\chi S B$ patterned after superconductivity and driven by a zero-range scalar and pseudoscalar quark-quark interaction, the cut-off $\Lambda_{N J L} \sim 631 \mathrm{MeV}$ of the effective chiral field theory provides an estimate for the momentum scale where the dynamical quark mass from the gap equation has fallen to the current quark mass. A more realistic treatment of color confinement is provided by the dual superconductor picture in the 't Hooft abelian gauge [2] where QCD monopoles generated by the abelian gauge fixing condense in the QCD vacuum confining quarks by the dual Meissner effect. Here the dynamical $\chi S B$ leads to a quark mass consistent with the QCD running whose monopole shape parameter $\Lambda_{m} \sim \Lambda_{Q C D} \sqrt{10} \sim 630 \mathrm{MeV}$ is close to $\Lambda_{N J L}$ [3]. At this momentum scale current quarks become the relevant degrees of freedom.

At momentum scale $\Lambda_{Q C D}$ chiral perturbation theory $(\chi \mathrm{PT}$ [4] ) allows incorporating systematically the chiral dynamics of QCD. Chiral field theory applies to scales from $\Lambda_{Q C D}$ up to a (presumed) chiral symmetry restoration scale $\Lambda_{\chi}=4 \pi f_{\pi}=1169 \mathrm{MeV}$ where $f_{\pi}=93$ $\mathrm{MeV}$ is the pion decay constant. In this scenario, inside hadrons at distances that are smaller than the confinement scale $\Lambda_{Q C D}^{-1}$, but larger than distances where perturbative QCD (pQCD) applies, quarks and Goldstone bosons are the effective degrees of freedom. In principle, chiral field theory dissolves a dynamical or constituent quark into a current quark and a cloud of virtual Goldstone bosons, but here only one layer of Goldstone bosons is included. Thus, the effective degrees of freedom and interactions of chiral field theory are consistent with those of chiral perturbation theory.

Chiral fluctuations of valence quarks inside hadrons, $q_{\uparrow, \downarrow} \rightarrow q_{\downarrow, \uparrow}+\left(q \bar{q}^{\prime}\right)_{0}$, into pseudoscalar mesons, $\left(q \bar{q}^{\prime}\right)_{0}$, of the $\mathrm{SU}(3)$ flavor octet of Goldstone bosons, were first applied to the spin problem of the proton in ref. [5]. It was shown that chiral dynamics can help one understand not only the reduction of the proton spin carried by the valence quarks from $\Delta \Sigma=1$ in the NQM to the experimental value of about $1 / 3$, but also the reduction of the axial vector

coupling constant $g_{A}^{(3)}$ from the NQM value $5 / 3$ to about $5 / 4$. It is well known that relativistic effects reduce the axial charge further, and this causes problems for the spin fractions in relativistic quark models [6] forcing the proton size parameter to a rather large value of about $1 \mathrm{fm}$. This problem will no longer occur when we replace the NQM by constraints from the $\mathrm{SU}(6)$ spin-flavor wave function of the proton. In addition, the violation of the Gottfried sum rule [7] $G S R=1 / 3+2(\bar{u}-\bar{d}) / 3$, which signals an isospin asymmetric quark sea in the proton, became plausible. The $\mathrm{SU}(3)$ symmetric chiral quark model explains several spin and sea quark observables of the proton, but not all of them. The data [8,9] call 
for $\mathrm{SU}(3)$ breaking because some of the spin fractions such as the ratio of triplet to octet spin fractions $\Delta_{3} / \Delta_{8}=5 / 3$ and the weak axial vector coupling constant of the nucleon,

$g_{A}^{(3)}=\mathcal{F}+\mathcal{D}$, disagree with experiments in the $\mathrm{SU}(3)$ symmetric case. In 10,11] the effects of $\mathrm{SU}(3)$ breaking were more systematically built into these chiral models and shown to lead to a remarkable further improvement of the spin and quark sea observables (that are integrated over Bjorken $\mathrm{x}$ ) in comparison with the data. It was also shown [10] that the $\eta^{\prime}$ meson proposed in 12 gives an almost negligible contribution to the spin fractions of the nucleon at the present level of experimental errors not only because of its large mass but also due to the small singlet chiral coupling constant. It is therefore ignored in the following, and this is consistent with the understanding that, due to the axial anomaly, the $\eta^{\prime}$ meson is not a genuine Goldstone boson.

A major objective here is to construct proton structure functions so that, when integrated over Bjorken $\mathrm{x}$, they reproduce the recent successful chiral field theory results for the spin and flavor fractions of the proton [10,6]. This goal can be achieved, but only upon neglecting helicity non-flip chiral transitions in the polarized splitting functions. The latter are also neglected in the unpolarized splitting functions, but this approximation is not nearly as critical.

Standard quark models like the nonrelativistic NQM or light-cone quark models are at scale $\Lambda_{Q C D}$, and it is not clear how these models should be evolved to the scale $\Lambda_{\chi}$ in the non-perturbative regime where initial quark distributions are needed. In [13] ratios of structure functions were found to be fairly insensitive to the uncertainties inherent in the evolution and were therefore recommended as a tool for testing quark models for missing physics. An example is the connection between the negative slope of the ratio of unpolarized structure functions, $F_{2}^{n} / F_{2}^{p}$, and a spin dependent attraction between the up and down quarks in the proton. In order to construct initial valence distributions, we turn to a more successful alternative which has recently been developed in [14], called BBS henceforth. This approach is based on quark counting rules or, more precisely, it implements the leading power-law behavior at $x \rightarrow 1$ of the helicity-dependent distributions that is known from the minimally connected perturbative diagrams in conjunction with constraints from the $\mathrm{SU}(6)$ proton spin-flavor wave function of the NQM. The initial parton distributions provided by BBS have been found surprisingly successful when evolved to the scale of the deep inelastic scattering (DIS) data from about $2 \mathrm{GeV}$ [15]. Therefore, this approach is adopted and used as a benchmark to assess chiral dynamics for its ability to generate intrinsic quark-antiquark excitations from initial valence quark distributions of the proton. We shall see that chiral field theory then predicts reasonable sea quark distributions.

The chiral field theory of parton distributions is described in Section II. The antiquark and polarized quark distributions are presented and discussed in Section III. Moments are given in Section IV and used in Section V to impose quark counting rules from pQCD on the valence part of the initial parton distributions.

\section{QUARK DISTRIBUTIONS FROM CHIRAL FIELD THEORY}

If the spontaneous chiral symmetry breakdown in the infrared regime of QCD is governed by chiral $S U(3)_{L} \times S U(3)_{R}$ transformations, then the effective interaction between the octet of Goldstone boson fields $\Phi_{i}$ involves the axial-vector coupling 


$$
\mathcal{L}_{i n t}=-\frac{g_{A}}{2 f_{\pi}} \sum_{i=1}^{8} \bar{q} \partial_{\mu} \gamma^{\mu} \gamma_{5} \lambda_{i} \Phi_{i} q
$$

which can flip the polarization of quarks: $q_{\downarrow} \rightarrow q_{\uparrow}+G B$, etc. In Eq. 1, the $\lambda_{i},(i=$ $1,2, \ldots, 8)$ are Gell-Mann's $\mathrm{SU}(3)$ flavor matrices, and $g_{A}$ is the dimensionless axial vector quark coupling constant that is taken to be 1 here, while

$$
g_{A}^{(3)}=\Delta u-\Delta d=\Delta_{3}=\mathcal{F}+\mathcal{D}=\left(G_{A} / G_{V}\right)_{n \rightarrow p},
$$

is the isotriplet axial vector coupling constant of the weak decay of the neutron, and $\Delta u, \Delta d$ and $\Delta s$ stand for the fraction of proton spin carried by the $\mathrm{u}, \mathrm{d}$ and $\mathrm{s}$ quarks, respectively. They are defined by the matrix elements of the singlet, triplet, octet axial vector currents, $A_{\mu}^{(i)}$ for $\mathrm{i}=0,3,8$ of the nucleon state at zero momentum transfer. It is also common to define the hypercharge or octet spin fraction $\Delta_{8}$ and the total proton spin $2 S_{z}=\Delta \Sigma$ in the infinite momentum frame as

$$
\Delta_{8}=\Delta u+\Delta d-2 \Delta s=3 \mathcal{F}-\mathcal{D}, \quad \Delta \Sigma=\Delta u+\Delta d+\Delta s .
$$

The spin fractions that we discussed so far are related to the first moments of the polarized structure functions $g_{1}^{p}(x), g_{1}^{n}(x)$ of the proton which have been measured in a series of SLAC (E142, E143, E154 [8]) and CERN (EMC, NMC, SMC [9]) deep inelastic lepton scattering experiments with increasingly sophisticated analyses based on pQCD.

Returning to chiral field theory that we use for a description of DIS structure functions of the proton, note that, despite the nonperturbative nature of the chiral symmetry breakdown, the interaction between quarks and Goldstone bosons is small enough for a perturbative expansion to apply.

Writing only the flavor dependence of the chiral interaction the SU(3) symmetric Eq. 11 has the matrix form

$$
\begin{gathered}
L_{i n t}=\frac{g_{A}}{2 f_{\pi}} \sum_{i=1}^{8} \bar{q} \lambda_{i} \Phi_{i} q \\
\frac{1}{\sqrt{2}} \sum_{i=1}^{8} \lambda_{i} \Phi_{i}=\left(\begin{array}{ccc}
\frac{1}{\sqrt{2}} \pi^{0}+\frac{1}{\sqrt{6}} \eta & \pi^{+} & K^{+} \\
\pi^{-} & -\frac{1}{\sqrt{2}} \pi^{0}+\frac{1}{\sqrt{6}} \eta & K^{0} \\
K^{-} & \bar{K}^{0} & -\frac{2}{\sqrt{6}} \eta
\end{array}\right) .
\end{gathered}
$$

From Eq. 11 the following probability densities $f\left(u_{\uparrow} \rightarrow \pi^{+}+d_{\downarrow}\right), \ldots$ for chiral fluctuations of quarks can be organized as coefficients in chiral reactions:

$$
\begin{aligned}
& u_{\uparrow} \rightarrow f_{u \rightarrow \pi^{+} d}\left(x_{\pi}, \mathbf{k}_{\perp}^{2}\right)\left(\pi^{+}+d_{\downarrow}\right)+f_{u \rightarrow \eta u} \frac{1}{6}\left(\eta+u_{\downarrow}\right)+f_{u \rightarrow \pi^{0} u} \frac{1}{2}\left(\pi^{0}+u_{\downarrow}\right) \\
& +f_{u \rightarrow K^{+}{ }_{s}}\left(K^{+}+s_{\downarrow}\right), \\
& d_{\uparrow} \rightarrow f_{d \rightarrow \pi^{-} u}\left(\pi^{-}+u_{\downarrow}\right)+f_{d \rightarrow \eta d} \frac{1}{6}\left(\eta+d_{\downarrow}\right)+f_{d \rightarrow \pi^{0} d} \frac{1}{2}\left(\pi^{0}+d_{\downarrow}\right) \\
& +f_{d \rightarrow K^{0} s}\left(K^{0}+s_{\downarrow}\right), \\
& s_{\uparrow} \rightarrow f_{s \rightarrow \eta s} \frac{2}{3}\left(\eta+s_{\downarrow}\right)+f_{s \rightarrow K^{-} u}\left(K^{-}+u_{\downarrow}\right)+f_{s \rightarrow \bar{K}^{0} d}\left(\bar{K}^{0}+d_{\downarrow}\right),
\end{aligned}
$$


and corresponding ones for the other initial quark helicity. After integrating over transverse momentum in the infinite momentum frame, the coefficients in Eq. 6 become the polarized (minus sign) and unpolarized (plus sign) chiral splitting functions,

$$
P_{G B / q}^{ \pm}(x)=\int d^{2} \mathbf{k}_{\perp} f_{q \rightarrow G B q^{\prime}}^{ \pm}\left(x, \mathbf{k}_{\perp}\right)
$$

that determine the probability for finding a Goldstone boson of mass $m_{G B}$ carrying the longitudinal momentum fraction $x_{G B}$ of the parent quark's momentum and a recoil quark $q^{\prime}$ with momentum fraction $1-x_{G B}$ for each fluctuation of Eq. 6 .

In (non-renormalizable) chiral field theory with cut-off $\Lambda_{\chi}$ of ref. [5], the unpolarized chiral splitting functions are

$$
P_{q \rightarrow q^{\prime}+G B}^{+}\left(x_{G B}\right)=\frac{g_{A}^{2}}{f_{\pi}^{2}} \frac{x_{G B}\left(m_{q}+m_{q^{\prime}}\right)^{2}}{32 \pi^{2}} \int_{-\Lambda_{\chi}^{2}}^{t_{\text {min }}} d t \frac{\left[\left(m_{q}-m_{q^{\prime}}\right)^{2}-t\right]}{\left(t-m_{G B}^{2}\right)^{2}},
$$

where $t=-\left[\mathbf{k}_{\perp}^{2}+x_{G B}\left[m_{q^{\prime}}^{2}-\left(1-x_{G B}\right) m_{q}^{2}\right]\right] /\left(1-x_{G B}\right)$ is the square of the Goldsone boson four-momentum. Next we obtain the polarized splitting functions from the unpolarized ones using that the latter contain the sum of helicity non-flip and flip probabilities, while the former contain the difference of these chiral probabilities. Since quarks are on their mass shell in the light-front dynamics used here, the axial vector quark-Goldstone boson interaction is equivalent to the simpler $\gamma_{5}$ interaction. Except for a common overall factor, the relevant unpolarized chiral transition probability is proportional to

$$
-\frac{1}{2} \operatorname{tr}\left[\left(\gamma \cdot p+m_{q}\right) \gamma_{5}\left(\gamma \cdot p^{\prime}+m_{q}^{\prime}\right) \gamma_{5}\right]=2\left(p p^{\prime}-m_{q} m_{q}^{\prime}\right)=\left(m_{q}-m_{q}^{\prime}\right)^{2}-k^{2},
$$

where $2 p p^{\prime}=m_{q}^{\prime 2}+m_{q}^{2}-k^{2}$. This is the numerator in Eq. 8 which can also be written as

$$
\frac{1}{1-x_{G B}}\left[\mathbf{k}_{\perp}^{2}+\left[m_{q^{\prime}}-\left(1-x_{G B}\right) m_{q}\right]^{2}\right]
$$

and has the following physical interpretation. Recall that the axial-vector quark-Goldstone boson coupling $\gamma_{\mu} \gamma_{5} k^{\mu}$ in Eq. 1 involves the spin raising and lowering operators $\sigma_{1} \pm i \sigma_{2}$ in a scalar product with the transverse momentum $\mathbf{k}_{\perp}$ of the recoil quark, which suggests that the $\mathbf{k}_{\perp}^{2}$ term in Eq. 10 represents the helicity-flip probability of the chiral fluctuation, while the longitudinal and time components $\gamma_{5} \gamma_{ \pm}$induce the non-spinflip probability which depends on the quark masses. This can be seen from the helicity non-flip probability

$$
\left|\bar{u}_{\uparrow}^{\prime} \gamma_{5} u_{\uparrow}\right|^{2}=\left|\bar{u}_{\downarrow}^{\prime} \gamma_{5} u_{\downarrow}\right|^{2} \sim\left(m_{q}^{\prime}-x^{\prime} m_{q}\right)^{2}, x^{\prime}=1-x_{G B},
$$

using light cone spinors and suppressing the spinor normalizations. Thus Eq. 11 identifies the mass term in Eq. 10 as the helicity non-flip chiral transition. Similarly, the helicity-flip probability is obtained from

$$
\left|\bar{u}_{\downarrow}^{\prime} \gamma_{5} u_{\uparrow}\right|^{2} \sim \mathbf{p}_{\perp}^{\prime 2}+x^{\prime 2} \mathbf{p}_{\perp}^{2}-x^{\prime} \mathbf{p}_{\perp}^{\prime} \cdot \mathbf{p}_{\perp}
$$

which, in frames where $\mathbf{p}_{\perp}=0$, reduces to $\mathbf{k}_{\perp}^{2}$, i.e. the net helicity flip probability generated by the chiral splitting process. In the nonrelativistic limit, where $\left|\mathbf{p}_{\perp}^{\prime}\right|<<m_{q^{\prime}},\left|\mathbf{p}_{\perp}\right|<<m_{q}$, clearly non-spinflip dominates over spinflip, while spinflip dominates at high momentum. 
The polarized splitting function $P^{-}$therefore has the same quark mass dependence as $P^{+}$, but involves the helicity flip probability with the opposite sign, i.e. has

$$
\frac{1}{1-x_{G B}}\left[-\mathbf{k}_{\perp}^{2}+\left[m_{q^{\prime}}-\left(1-x_{G B}\right) m_{q}\right]^{2}\right]
$$

in its numerator, which agrees with ref. [16.

The $q \rightarrow q^{\prime} G B$ splitting functions are expected to satisfy number and momentum sum rules [17]. For example, if the quark $q$ is accompanied by two spectator quarks of a nucleon which splits (virtually) into a Goldstone boson and an octet baryon $B$ containing the recoil quark $q^{\prime}$ along with the spectator quark pair, then the splitting function in Eq. 13 becomes the hadronic $f_{G B / N}\left(x_{G B}\right)$ for $N \rightarrow B+G B$, which equals $f_{B / N}\left(1-x_{G B}\right)$, since the recoil quark $q^{\prime}$ has longitudinal momentum fraction $1-x_{G B}$. Therefore, there are equal numbers of Goldstone bosons emitted by the nucleon, $\langle n\rangle_{G B / N}$, and virtual baryons accompanying them, $\langle n\rangle_{B / N}$. This symmetry is violated by Eq. 8 of the model [5]. Furthermore, momentum conservation holds, viz. $\left\langle x_{G B}\right\rangle_{G B / N}+\left\langle x_{B}\right\rangle_{B / N}=\langle n\rangle_{G B / N}$. In order to satisfy the symmetry and momentum conservation laws, we follow ref. [17] and replace the cut-off in Eq. \& by a vertex function $G$ in the splitting function integrand

$$
f_{q \rightarrow G B q^{\prime}}^{ \pm}\left(x_{G B}, \mathbf{k}_{\perp}^{2}\right)=\frac{g_{G B q q^{\prime}}^{2}}{16 \pi^{3}} \frac{\left|G_{G B q q^{\prime}}\left(x_{G B}, \mathbf{k}_{\perp}^{2}\right)\right|^{2}}{x_{G B}\left(1-x_{G B}\right)} \frac{\left(\left(1-x_{G B}\right) m_{q}-m_{q^{\prime}}\right)^{2} \pm \mathbf{k}_{\perp}^{2}}{\left(1-x_{G B}\right)\left(m_{q}^{2}-M_{G B q^{\prime}}^{2}\right)^{2}}
$$

where $\mathbf{k}_{\perp}$ is the transverse momentum of the recoil quark $q^{\prime}$ whose longitudinal momentum fraction of the parent quark's is $1-x_{G B}$. Note that

$$
f_{u \rightarrow u \pi^{0}}^{ \pm}=f_{u \rightarrow d \pi^{+}}^{ \pm}=f_{d \rightarrow d \pi^{0}}^{ \pm}=f_{d \rightarrow u \pi^{-}}^{ \pm} .
$$

The vertex function $G_{G B q q^{\prime}}$ in Eq. 13 takes into account the extended structure of the Goldstone boson-quark system. It is taken here to depend on the inverse of the same covariant propagator $\left(m_{q}^{2}-M_{G B q^{\prime}}^{2}\right)^{-1}$ that occurs in the splitting function in Eq. 13,

$$
G_{G B q q^{\prime}}\left(x_{G B}, \mathbf{k}_{\perp}^{2}\right)=\exp \left(\frac{m_{q}^{2}-M_{G B q^{\prime}}^{2}\left(x_{G B}, \mathbf{k}_{\perp}^{2}\right)}{2 \Lambda^{2}}\right)
$$

with the invariant mass squared of the quark-Goldstone boson system $G B+q^{\prime}$

$$
M_{G B q^{\prime}}^{2}\left(x_{G B}, \mathbf{k}_{\perp}^{2}\right)=\frac{m_{G B}^{2}+\mathbf{k}_{\perp}^{2}}{x_{G B}}+\frac{m_{q^{\prime}}^{2}+\mathbf{k}_{\perp}^{2}}{1-x_{G B}} .
$$

Such models satisfy the symmetry constraint mentioned above.

The parameter $\Lambda$ in Eq. 14 controls the size of the Goldstone boson-quark system so that $2 / \Lambda \sim<r^{2}>_{G B q^{\prime}}^{1 / 2} \leq \frac{1}{3} \mathrm{fm}$. If we relate the monopole shape of $m_{q}\left(p^{2}\right)$ mentioned in the Introduction to a Gaussian shape, then $\Lambda \sim 2 \sqrt{5} \Lambda_{Q C D} / 0.78 \sim 1.14 \mathrm{GeV}$ is obtained. Such hadronic descriptions of meson clouds of baryons are currently studied by many groups. Since the vertex-times-propagator form of the splitting functions in Eq. 13 admits a wave function interpretation for the virtual hadronic process $N \rightarrow B+G B$, a light-front wavefunction parametrization has been adopted in ref. [18 for $N \rightarrow \Lambda+K$. 
The pion-quark coupling constant in Eq. 13 may be obtained from the GoldbergerTreiman relation $(\mathrm{GTR})$

$$
g_{\pi N N}=g_{A}^{(3)} \frac{m_{N}}{f_{\pi}}
$$

where $f_{\pi} \sim 93 \mathrm{MeV}$ is the pion decay constant, in conjunction with the NQM coupling constant relation

$$
g_{\pi q q^{\prime}}=\frac{3}{5} g_{\pi N N}
$$

If a constituent quark mass instead of the nucleon mass is used in a GTR for constituent quarks as in Eq. 8,

$$
g_{\pi q q^{\prime}}=g_{A} \frac{m_{q}+m_{q^{\prime}}}{2 f_{\pi}}
$$

which is common in chiral quark models at scale $\Lambda_{Q C D}$, then the factor $3 / 5$ in Eq. 17 is replaced by $g_{A} / 3 g_{A}^{(3)}$, where $g_{A}$ is the quark axial-vector coupling constant often taken to be $3 / 4$ or 1 . Since the factor $3 / 5$ in Eq. 17 is scale (and model) dependent, we set $g_{\pi q q^{\prime}}=\alpha_{\chi} g_{A}^{(3)} m_{N} / f_{\pi}$ and vary the chiral strength parameter $\alpha_{\chi}$ within the limits $\frac{1}{5} \leq \alpha_{\chi} \leq \frac{3}{5}$. In the $\mathrm{SU}(3)$ symmetric case $f_{K} \sim f_{\pi}$ for the kaons, and we set $g_{K q q^{\prime}}=g_{\pi q q^{\prime}}$.

The integral over Bjorken $\mathrm{x}$ of the splitting function

$$
P_{G B / q}=\left|\alpha_{G B / Q}\right|^{2}=\int_{0}^{1} d x \int d^{2} \mathbf{k}_{\perp} f_{q \rightarrow G B q^{\prime}}^{+},
$$

is the probability of finding a virtual Goldstone boson $G B$ in the initial quark $q$. This in turn is related to the Fockspace expansion of the dressed quarks (in light front field theory where it is well defined), e.g.

$$
|U\rangle=\sqrt{Z}\left|u_{0}\right\rangle+\alpha_{\pi / U}\left|d \pi^{+}\right\rangle+\frac{1}{2} \alpha_{\pi / U}\left|u \pi^{0}\right\rangle+\frac{1}{6} \alpha_{\eta / U}|u \eta\rangle+\alpha_{K / U}\left|s K^{+}\right\rangle+\ldots
$$

where the factors $p_{\pi^{0}}=1 / 2$ for the neutral pion, $p_{\eta}=1 / 6$ and $p_{\pi^{ \pm}}=1=p_{K^{ \pm}}=1$ come from the flavor dependence in Eq. 5, $Z=1-P_{u}$ is the wave function renormalization constant and all masses and coupling constants are renormalized quantities.

From the $\mathrm{u}$ and $\mathrm{d}$ quark lines in Eq. 6 the total meson emission probability $P_{u}$ of the $\mathrm{u}$ quark is given to first order in the Goldstone fluctuations by

$$
P_{u}=\int_{0}^{1} d x \int d^{2} \mathbf{k}_{\perp}\left[\frac{1}{2} f_{u \rightarrow u \pi^{0}}^{+}+\frac{1}{6} f_{u \rightarrow u \eta}^{+}+f_{u \rightarrow d \pi^{+}}^{+}+f_{u \rightarrow s K^{+}}^{+}\right]=\sum_{q, m} p_{m} \int_{0}^{1} d x P_{u \rightarrow q m}^{+},
$$

while the total strange quark probability density

$$
P_{s}=\int_{0}^{1} d x \int d^{2} \mathbf{k}_{\perp}\left[\frac{2}{3} f_{s \rightarrow s \eta}^{+}+f_{s \rightarrow u K^{-}}^{+}+f_{s \rightarrow d \bar{K}^{0}}^{+}\right]
$$

can be read off the s quark line in Eq. 6 and total meson emission from the d quark is equal to that from the u quark, $P_{d}=P_{u}$. 
The proton's probability composition law [5, 10, 11, 6]

$$
\begin{gathered}
\left(1-P_{u}\right)\left(u_{\uparrow}^{0} \hat{u}_{\uparrow}+u_{\downarrow}^{0} \hat{u}_{\downarrow}+d_{\uparrow}^{0} \hat{d}_{\uparrow}+d_{\downarrow}^{0} \hat{d}_{\downarrow}\right) \\
+u_{\uparrow}^{0} \sum_{q, m} p_{m} P_{u_{\uparrow} \rightarrow q_{\downarrow} m}^{+}+u_{\downarrow}^{0} \sum_{q, m} p_{m} P_{u_{\downarrow} \rightarrow q_{\uparrow} m}^{+}+d_{\uparrow}^{0} \sum_{q, m} p_{m} P_{d_{\uparrow} \rightarrow q_{\downarrow} m}^{+}+d_{\downarrow}^{0} \sum_{q, m} p_{m} P_{d_{\downarrow} \rightarrow q_{\uparrow} m}^{+}
\end{gathered}
$$

is a useful tool in conjunction with Eq. 6 for keeping track of chiral fluctuations from valence quark distributions $q^{0}(x)$. The latter will be addressed in Sect.V.

The splitting of quarks into a Goldstone boson and a recoil quark corresponds to a factorization of DIS structure functions that is equivalent to a convolution of quark distributions with splitting functions at a scale to be discussed in Sect.III. From Eqs. 6, 23 the unpolarized quark distributions off valence quark distributions, $q^{0}(x)$, and up to second order chiral fluctuations, are given by convolution integrals of the general form

$$
\begin{gathered}
P^{+} \otimes q^{0}=\int_{x}^{1} \frac{d x_{1}}{x_{1}} P_{q \rightarrow q^{\prime} G B}^{+}\left(\frac{x}{x_{1}}\right) q^{0}\left(x_{1}\right), \\
q \otimes P^{+} \otimes q^{0}=\int_{x}^{1} \frac{d x_{1}}{x_{1}} q_{G B}\left(\frac{x}{x_{1}}\right) \int_{x_{1}}^{1} \frac{d x_{2}}{x_{2}} P_{q \rightarrow q^{\prime} G B}^{+}\left(\frac{x_{1}}{x_{2}}\right) q^{0}\left(x_{2}\right),
\end{gathered}
$$

so that

$$
\begin{aligned}
& u(x)=Z u^{0}+P_{d \rightarrow u \pi^{-}}^{+} \otimes d^{0}+\left(\frac{1}{2} P_{u \rightarrow u \pi^{0}}^{+}+\frac{1}{6} P_{u \rightarrow u \eta}^{+}\right) \otimes u^{0}+u_{\pi^{+}}^{0} \otimes P_{u \rightarrow d \pi^{+}}^{+} \otimes u^{0} \\
& +u_{K^{+}}^{0} \otimes P_{u \rightarrow s K^{+}}^{+} \otimes u^{0}+\frac{1}{4} u_{\pi^{0}}^{0} \otimes P_{q \rightarrow q \pi^{0}}^{+} \otimes\left(u^{0}+d^{0}\right)+\frac{1}{36} u_{\eta}^{0} \otimes P_{q \rightarrow q \eta}^{+} \otimes\left(u^{0}+d^{0}\right) \\
& +\frac{1}{6} u_{\pi^{0}, \eta}^{0} \otimes P_{q \rightarrow q \pi^{0}, \eta}^{+} \otimes\left(u^{0}-d^{0}\right), \\
& \begin{array}{c}
d(x)=Z d^{0}+P_{u \rightarrow d \pi^{+}}^{+} \otimes u^{0}+\left(\frac{1}{2} P_{d \rightarrow d \pi^{0}}^{+}+\frac{1}{6} P_{d \rightarrow d \eta}^{+}\right) \otimes d^{0}+d_{\pi^{-}}^{0} \otimes P_{d \rightarrow u \pi^{-}}^{+} \otimes d^{0} \\
+d_{K^{0}}^{0} \otimes P_{d \rightarrow s K^{0}}^{+} \otimes d^{0}+\frac{1}{4} d_{\pi^{0}}^{0} \otimes P_{q \rightarrow q \pi^{0}}^{+} \otimes\left(u^{0}+d^{0}\right)+\frac{1}{36} d_{\eta}^{0} \otimes P_{q \rightarrow q \eta}^{+} \otimes\left(u^{0}+d^{0}\right) \\
-\frac{1}{6} u_{\pi^{0}, \eta}^{0} \otimes P_{q \rightarrow q \pi^{0}, \eta}^{+} \otimes\left(u^{0}-d^{0}\right), \\
s=P_{u \rightarrow s K^{+}}^{+} \otimes u^{0}+P_{d \rightarrow s K^{0}}^{+} \otimes d^{0}+\frac{4}{9} s_{\eta}^{0} \otimes P_{q \rightarrow q \eta}^{+} \otimes\left(u^{0}+d^{0}\right) .
\end{array}
\end{aligned}
$$

Here the $q_{G B}^{0}$ are the valence quark distributions of the respective Goldstone boson. 
Moreover, the $u$ (or $d$ ) amplitudes from the $\pi^{0}$ and $\eta$ meson exchanges add coherently to the total antiquark probability amplitude of the proton. We know from ref. [5, 10,11] that these interference amplitudes, i.e. the last terms in Eq. 24,25, cannot be neglected. Here we have approximated the $\left(\pi^{0}, \eta\right)$ interference terms by using equal $\pi^{0}$ and $\eta$ splitting functions and quark distributions $q_{\pi^{0}} \approx q_{\eta} \approx q$ in these mesons so that

$$
\begin{aligned}
\int_{x}^{1} \frac{d x_{1}}{x_{1}}\left[q_{\pi^{0}}\left(x_{1}\right) q_{\eta}\left(x_{1}\right)\right]^{1 / 2} & \int_{x_{1}}^{1}\left[P_{q \rightarrow q\left(\pi^{0}\right)}^{+}\left(\frac{x_{2}}{x_{1}}\right) P_{q \rightarrow q(\eta)}^{+}\left(\frac{x_{2}}{x_{1}}\right)\right]^{1 / 2} q^{0}\left(\frac{x}{x_{2}}\right) \frac{d x_{2}}{x_{2}} \\
& \sim \int_{x}^{1} \frac{d x_{1}}{x_{1}} q\left(x_{1}\right) \int_{x_{1}}^{1} P_{q \rightarrow q\left(\pi^{0}, \eta\right)}^{+}\left(\frac{x_{2}}{x_{1}}\right) q^{0}\left(\frac{x}{x_{2}}\right) \frac{d x_{2}}{x_{2}}
\end{aligned}
$$

takes the form of a conventional parton distribution.

\section{ANTIQUARK AND SPIN DISTRIBUTIONS}

In chiral dynamics, antiquarks originate only from the Goldstone bosons via their quarkantiquark composition, viz.

$$
\left|\pi^{0}\right\rangle=\frac{1}{\sqrt{2}}(\bar{u} u-\bar{d} d),|\eta\rangle=\frac{1}{\sqrt{6}}(\bar{u} u+\bar{d} d-2 \bar{s} s),\left|K^{+}\right\rangle=u \bar{s},
$$

etc. Therefore, antiquarks are unpolarized. Small antiquark polarizations are consistent with the most recent SMC data [9] so that we expect only small corrections if we use $\bar{u}_{\uparrow}=\bar{u}_{\downarrow}$ in the spin fractions $\Delta u=u_{\uparrow}-u_{\downarrow}+\bar{u}_{\uparrow}-\bar{u}_{\downarrow}$, etc., i.e. $\Delta s=\Delta s_{\text {sea }}, \Delta \bar{u}=\Delta \bar{d}=\Delta \bar{s}=0$.

An antiquark in a Goldstone boson which is struck by the virtual photon in DIS is linked to a valence quark of the proton by Goldstone boson exchange. Therefore, antiquarks appear

only in second order of chiral fluctuations. Thus, from Eqs. 6,23 the antiquark fractions of the proton are given by double convolution integrals of the general form $\bar{q} \otimes P_{q \rightarrow q G B} \otimes q^{0}$,

$$
\begin{gathered}
\bar{u}(x)=\bar{u}_{\pi^{-}}^{0} \otimes P_{d \rightarrow u \pi^{-}}^{+} \otimes d^{0}+\frac{1}{4} \bar{u}_{\pi^{0}}^{0} \otimes P_{q \rightarrow q \pi^{0}}^{+} \otimes\left(u^{0}+d^{0}\right)+\frac{1}{36} \bar{u}_{\eta}^{0} \otimes P_{q \rightarrow q \eta}^{+} \otimes\left(u^{0}+d^{0}\right) \\
+\frac{1}{6} \bar{u}_{\pi^{0}, \eta}^{0} \otimes P_{q \rightarrow q \pi^{0}, \eta}^{+} \otimes\left(u^{0}-d^{0}\right) \\
\bar{d}(x)=\bar{d}_{\pi^{+}}^{0} \otimes P_{u \rightarrow d \pi^{+}}^{+} \otimes u^{0}+\frac{1}{4} \bar{d}_{\pi^{0}}^{0} \otimes P_{q \rightarrow q \pi^{0}}^{+} \otimes\left(u^{0}+d^{0}\right)+\frac{1}{36} \bar{d}_{\eta}^{0} \otimes P_{q \rightarrow q \eta}^{+} \otimes\left(u^{0}+d^{0}\right) \\
-\frac{1}{6} \bar{d}_{\pi^{0}, \eta}^{0} \otimes P_{q \rightarrow q \pi^{0}, \eta}^{+} \otimes\left(u^{0}-d^{0}\right), \\
\bar{s}=\bar{s}_{K^{0}}^{0} \otimes P_{d \rightarrow s K^{0}}^{+} \otimes \\
d^{0}+\bar{s}_{K^{+}}^{0} \otimes P_{u \rightarrow s K^{+}}^{+} \otimes u^{0}+\frac{4}{9} \bar{s}_{\eta}^{0} \otimes P_{q \rightarrow q \eta}^{+} \otimes\left(u^{0}+d^{0}\right) .
\end{gathered}
$$

Again, we have approximated the $\left(\pi^{0}, \eta\right)$ interference terms, the last expression in Eqs. 30,31, by using equal $\pi^{0}$ and $\eta$ splitting functions and the same antiquark distributions in these mesons. 
The $\bar{s}(x)$ and $s(x)$ distributions of Eqs. 26, 32, respectively, are no longer equal unless the $\mathrm{s}$ valence antiquark distributions in the $\mathrm{K}$ mesons, $\bar{s}_{K^{0}, K^{+}}^{0} \sim \delta(1-x)$, are approximated by the elastic limit. When spectator quarks are included in addition, but second order chiral fluctuations are neglected, then one recovers from Eq. 26 the strange quark distribution, $s(x)$, of some hadronic models [17,18].

Upon generalizing the formalism of [10 also to the polarized quark distributions, the probabilities displayed in Eq. 6 and Eq. 23 yield

$$
q_{\uparrow}(x)=\left(1-P_{q}\right) q_{\uparrow}^{0}(x)+\sum_{m, q^{\prime}} p_{m} P_{q^{\prime} \rightarrow q m}^{+} \otimes q_{\downarrow}^{00}+\ldots,
$$

where the constants $p_{m}$ are defined below Eq. 20 and $m$ denotes a Goldstone boson. The corresponding result holds for the other quark helicity. The ellipses in Eq. 33 denote double convolution terms with $q_{\uparrow}^{0}$ from a Goldstone boson $m$ that cancel in $q_{\uparrow}-q_{\downarrow}$. The opposite quark helicity on the rhs of Eq. 33 means that helicity non-flip chiral transitions are neglected and implies the negative sign of all chiral contributions to the spin distributions

$$
\Delta q(x)=\left(1-P_{q}\right) \Delta q^{0}(x)-\sum_{m, q^{\prime}} p_{m} P_{q^{\prime} \rightarrow q m}^{+} \otimes \Delta q^{\prime 0} .
$$

The general result involves the polarized splitting functions

$$
P_{q \rightarrow q^{\prime} G B}^{-}(y)=\int d^{2} \mathbf{k}_{\perp} f_{q \rightarrow q^{\prime} G B}^{-}\left(y, \mathbf{k}_{\perp}\right)
$$

and has the same form as Eq. 34,

$$
\begin{gathered}
\Delta u(x)=\left(1-P_{u}\right) \Delta u^{0}(x)+\int_{x}^{1} \frac{d y}{y}\left[\Delta u^{0}(y)\left(\frac{1}{2} P_{u \rightarrow u \pi^{0}}^{-}+\frac{1}{6} P_{u \rightarrow u \eta}^{-}\right)+\Delta d^{0}(y) P_{d \rightarrow u \pi^{-}}^{-}\right], \\
\Delta d(x)=\left(1-P_{d}\right) \Delta d^{0}(x)+\int_{x}^{1} \frac{d y}{y}\left[\Delta d^{0}(y)\left(\frac{1}{2} P_{d \rightarrow d \pi^{0}}^{-}+\frac{1}{6} P_{d \rightarrow d \eta}^{-}\right)+\Delta u^{0}(y) P_{u \rightarrow d \pi^{+}}^{-}\right], \\
\Delta s(x)=\int_{x}^{1} \frac{d y}{y}\left[\Delta d^{0}(y) P_{d \rightarrow s K^{0}}^{-}\left(\frac{x}{y}\right)+\Delta u^{0}(y) P_{u \rightarrow s K^{+}}^{-}\left(\frac{x}{y}\right)\right]
\end{gathered}
$$

except for the replacement of $-P^{+}$by the corresponding polarized splitting function $P^{-}$.

Due to the minus sign in the $\mathbf{k}_{\perp}^{2}$ term of the polarized splitting functions in Eq. 13 which corresponds to the subtracted terms in Eq.34, the general reduction of $\Delta q^{0}$ by chiral fluctuations in lowest order is the crucial property responsible for the success of chiral field theory for the proton spin fractions. A comparison of Eq. 34 with Eqs.36.37, 38, shows that only the unpolarized splitting function occurs in the polarized and unpolarized spin-flavor fractions of refs. [5:10 [12, 6], and the negative sign of $P^{-} \approx-P^{+}$is critical for the reductions of the spin fractions by the chiral fluctuations and the remarkable success of these models. Thus, when these $\Delta q(x)$ are integrated over Bjorken $\mathrm{x}$, the lowest moments (which factorize into products of moments as we discuss in the next section) reproduce precisely the structure of the results for the spin fractions [10,6] 


$$
\begin{array}{r}
\Delta u=(1-P) \Delta u^{0}-\left(\frac{2}{3} \Delta u^{0}+\Delta d^{0}\right) a\left(1+\frac{\epsilon}{\sqrt{3}}\right)^{2} \\
\Delta d=(1-P) \Delta d^{0}-\left(\frac{2}{3} \Delta d^{0}+\Delta u^{0}\right) a\left(1+\frac{\epsilon}{\sqrt{3}}\right)^{2} \\
\Delta s=-\left(\frac{2}{3} \Delta d^{0}+\Delta u^{0}\right) a\left(1-\frac{2 \epsilon}{\sqrt{3}}\right)^{2} .
\end{array}
$$

The pure spin flip approximation is valid at high quark momentum or, upon comparing $P^{-}=-P^{+}$of Eq. 34 with the numerator of the splitting functions in Eq. 13, when the quark masses are negligible compared to the transverse quark momentum $<|\mathbf{k}|_{\perp}>$, i.e. current quarks are the relevant degrees of freedom. The factor $2 / 3$ in Eq. 39 comes from adding $\frac{1}{2}+\frac{1}{6}$ in Eqs. 36, 37 using the same splitting function for the $\pi^{0}$ and $\eta$ mesons because the $\mathrm{SU}(3)$ breaking along the $\lambda_{8}$ direction (which is the same as for the standard baryon and meson mass splittings) does not suppress the $\eta$ contribution. In particular, taking the elastic limit for the quark distributions in the mesons, $q\left(x_{2}\right) \sim \delta\left(1-x_{2}\right)$, as well as the elastic-limit delta function for the valence quark distributions $q^{0}\left(x_{1}\right)$, but keeping all second order chiral fluctuations, yields all our previous integrated spin-flavor fractions [10,6] and Eq. 39 in particular. Comparing the unpolarized quark and antiquark fractions already yields the identification

$$
a\left(1+\frac{\epsilon}{\sqrt{3}}\right)^{2}=\int_{0}^{1} d x P_{u \rightarrow d \pi^{+}}^{+}, a\left(1-\frac{2 \epsilon}{\sqrt{3}}\right)^{2}=\int_{0}^{1} d x P_{u \rightarrow s K^{+}}^{+},
$$

where $a$ is the chiral coupling strength and $\epsilon$ the SU(3) breaking parameter of [10,6]. For other chiral models such as [11,12] similar identifications can be made.

This detailed comparison indicates that the success of these chiral models can be attributed to parametrizations that rely on pure helicity flip chiral transitions. Since the helicity non-flip chiral transition probabilities depend on the quark masses, these fluctuations are negligible only when the quark masses are small compared to the relevant transverse momentum range. This is not the case for constituent quarks. Therefore, chiral quark models for the spin fractions have to include helicity non-flip fluctuations. When this is done as in ref. [16] then the spin fractions disagree with the data.

\section{MOMENTS}

The proton spin observables $\Delta q$ are the lowest moments of, or integrals over, the polarized structure functions. Also, the constraint imposed on the quark distributions by the momentum involves the first moment $\int_{0}^{1} x q(x) d x$ of the quark distributions. Therefore, in the context of DIS and chiral dynamics, it is mandatory to consider moments of convolutions of quarks or antiquarks with splitting functions.

It is straightforward to verify that the integral of a convolution, e. g.

$$
\int_{0}^{1} \bar{q} \otimes P \otimes q f(x) d x=\int_{0}^{1} \bar{q}(x) f(x) d x \cdot \int_{0}^{1} P(x) f(x) d x \cdot \int_{0}^{1} q(x) f(x) d x
$$


factorizes provided $f$ is a multiplicative function, i.e. $f(x y)=f(x) f(y)$, such as the power law $x^{n}$ that defines the n'th moment. In other words, the moment of a convolution is the product of the moments of the components of the convolution.

Applying this general observation to the quark, antiquark and spin distributions of Eqs. 24,26, 30, 32, 36, 37,38 yields for the moments $q_{n} \equiv \int_{0}^{1} x^{n} q(x) d x$ the following relations

$$
\begin{gathered}
u_{n}=a_{n} u_{n}^{0}+b_{n} d_{n}^{0}, \quad d_{n}=a_{n} d_{n}^{0}+b_{n} u_{n}^{0}, \\
s_{n}=P_{u \rightarrow s K^{+}}^{+, n} u_{n}^{0}+P_{d \rightarrow s K^{0}}^{+, n} d_{n}^{0}+\frac{4}{9} s_{\eta, n}^{0} P_{q \rightarrow q \eta}^{+, n}\left(u_{n}^{0}+d_{n}^{0}\right), \\
\bar{u}_{n}=\bar{u}_{\pi-, n}^{0} P_{d \rightarrow u \pi^{-}}^{+, n} d_{n}^{0}+\frac{1}{4} \bar{u}_{\pi^{0}, n}^{0} P_{\pi^{0}}^{+, n}\left(u_{n}^{0}+d_{n}^{0}\right)+\frac{1}{36} \bar{u}_{\eta, n}^{0} P_{\eta}^{+, n}\left(u_{n}^{0}+d_{n}^{0}\right)+\frac{1}{6} \bar{u}_{n}^{0} P^{+, n}\left(u_{n}^{0}-d_{n}^{0}\right), \\
\bar{s}_{n}=\bar{s}_{K^{+}, n}^{0} P_{u \rightarrow s K^{+}}^{+, n} u_{n}^{0}+\bar{s}_{K^{0}, n}^{0} P_{d \rightarrow s K^{0}}^{+, n} d_{n}^{0}+\frac{4}{9} \bar{s}_{\eta, n}^{0} P_{q \rightarrow q \eta}^{+, n}\left(u_{n}^{0}+d_{n}^{0}\right),
\end{gathered}
$$

where the $P^{+, n}$ are the moments of the unpolarized splitting functions and the $q_{n}^{0}, \bar{s}_{n}^{0}$ are those of the proton and $\mathrm{K}$ meson valence quark distributions. The coefficients $a_{n}, b_{n}$ are given by

$$
\begin{gathered}
a_{n}=Z+\frac{1}{2} P_{\pi^{0}}^{+, n}+\frac{1}{6} P_{\eta}^{+, n}+u_{\pi^{+}, n}^{0} P_{u \rightarrow d \pi^{+}}^{+, n} \\
+\frac{1}{4} u_{\pi^{0}, n}^{0} P_{\pi^{0}}^{+, n}+\frac{1}{36} u_{\eta, n}^{0} P_{\eta}^{+, n}+\frac{1}{6} u_{n}^{0} P^{+, n}, \\
b_{n}=P_{d \rightarrow u \pi^{-}}^{+, n}+\frac{1}{4} u_{\pi^{0}, n}^{0} P_{\pi^{0}}^{+, n}+\frac{1}{36} u_{\eta, n}^{0} P_{\eta}^{+, n}-\frac{1}{6} u_{n}^{0} P^{+, n} .
\end{gathered}
$$

Similarly, from the $\Delta q$ in Eq. 38 the spin dependent moments are obtained as

$$
\begin{gathered}
\Delta u_{n}=\left(1-P_{u}\right) \Delta u_{n}^{0}+\left[\frac{1}{2} P_{\pi^{0}}^{-, n}+\frac{1}{6} P_{\eta}^{-, n}\right] \Delta u_{n}^{0}+P_{d \rightarrow u \pi^{-}}^{-, n} \Delta d_{n}^{0}, \\
\Delta d_{n}=\left(1-P_{d}\right) \Delta d_{n}^{0}+\left[\frac{1}{2} P_{\pi^{0}}^{-, n}+\frac{1}{6} P_{\eta}^{-, n}\right] \Delta d_{n}^{0}+P_{u \rightarrow d \pi^{+}}^{-, n} \Delta u_{n}^{0}, \\
\Delta s_{n}=P_{u \rightarrow s K^{+}}^{-, n} \Delta u_{n}^{0}+P_{d \rightarrow s K^{0}}^{-, n} \Delta d_{n}^{0}
\end{gathered}
$$

to be compared with Eq. 39 for $n=0$.

As a consequence of the factorization of moments of convolutions, the equality of the integrated $\bar{s}=s$ of the proton follows immediately from Eqs. 42,43 using $\bar{s}_{0}^{0}=1$ for the valence $\mathrm{s}$ antiquark fraction in the $\mathrm{K}$ mesons. In order to avoid more parameters and complications we shall use the elastic delta function approximation for the valence quarks and antiquarks in the Goldstone bosons in the numerical evaluations. 


\section{QCD CONSTRAINTS AND RESULTS}

There are numerous attempts to describe DIS structure functions based on quark models. These quark models provide initial quark distributions at the scale $\Lambda_{Q C D}$ as a rule. For the NQM [19] and a light-cone quark model (LCQM [13]) the up quark distributions are exhibited in Fig.1. The subsequent $Q^{2}$-evolution of structure functions follows from perturbative QCD, but relies on such initial shapes which contain the non-perturbative aspects of the bound state. The low-energy scale of a quark model is often treated as an adjustable parameter which is usually too small for a perturbative evolution to be valid. It is fair to say that, even with chiral fluctuations, quark models fall short of explaining the deep inelastic structure functions and the spin of the proton [16], quite apart from rather significant uncertainties in their evolution from $\Lambda_{Q C D}$ to about $\Lambda_{\chi}$.

The success of chiral fraction models based on pure helicity flip chiral fluctuations suggests that we construct initial quark distributions for the case where current quarks are the relevant degrees of freedom. Upon comparing with the momentum dependence of the dynamical quark mass discussed in the Introduction, the relevant momentum scale is about $\frac{2}{3} \mathrm{GeV}$ to $\Lambda_{\chi}$ rather than $\Lambda_{Q C D}$ of the NQM. It is important to realize in this context that the use of the spin fractions $\Delta q^{0}$ from the spin-flavor proton wave function of the NQM in Eq. 39 is not only justified at scale $\Lambda_{Q C D}$ but at $\Lambda_{\chi}$ as well because the (for $\Delta s^{0}=0$ ) equivalent isotriplet and octet axial charges $g_{A}^{(3)}, g_{A}^{(8)}$ are conserved in the chiral limit and scale independent.

Instead of the NQM we use the BBS approach to construct valence quark distributions. In contrast to BBS, we impose the quark counting rules directly on the valence quark distributions. There are no parametrizations for strange quarks or antiquarks which we expect to be generated by chiral fluctuations. It is an immediate consequence that initial parton distributions where the strange quarks contribute nothing (for some examples, see [20]) are ruled out by chiral field theory.

The constraints

$$
u_{0}^{0}=\int_{0}^{1} u^{0}(x) d x=2, \quad d_{0}^{0}=\int_{0}^{1} d^{0}(x) d x=1
$$

on valence quark numbers are characteristic features of quark models, such as the nonrelativistic NQM and light cone quark models. Moreover, the quark distributions satisfy $q^{0} \rightarrow 0$ as $x \rightarrow 0$ in these models. Hence for illustration purposes only, we parametrize such valence quark distributions $q^{0}(x)$ in terms of the shapes $x^{\alpha}(1-x)^{\beta}$ with a common positive exponent $\alpha$ and $\beta=3$, or 5 . As a consequence of the positive exponent, fewer non-leading terms are needed, which saves more than half of BBS's parameters, viz.

$$
\begin{gathered}
u_{\uparrow}^{0}(x)=A x^{\alpha}(1-x)^{3}, \quad u_{\downarrow}^{0}(x)=C x^{\alpha}(1-x)^{5}, \\
d_{\uparrow}^{0}(x)=\frac{A}{5} x^{\alpha}(1-x)^{3}, \quad d_{\downarrow}^{0}(x)=C^{\prime} x^{\alpha}(1-x)^{5},
\end{gathered}
$$

where the anti-parallel helicity quark distributions are suppressed by the factor $(1-x)^{2}$ and the exponent $\beta=3$ for $u_{\uparrow}^{0}$ and $d_{\uparrow}^{0}$ reflects the quark counting rule for the proton that guarantees the correct falloff of the leading form factors with $q^{2}$. The choice $A / 5$ for the 
leading coefficient of $d_{\uparrow}^{0}$ in Eq. 50 is motivated by the SU(6) structure of the NQM and its valence quark distributions for large $x$. When the other ratios of coefficients are also chosen as in the $\mathrm{SU}(6)$ spin-flavor wave function of the proton (cp. Eq.13 of [10]),

$$
C=\frac{A}{5}, C^{\prime}=\frac{2}{5} A
$$

then we obtain

$$
A=g_{A}^{(3)} \Pi_{j=1}^{6}(j+\alpha) / 6^{3}, \quad \alpha=\left[\frac{161}{4}+\frac{36 \Delta d^{0}}{g_{A}^{(3)}}\right]^{1 / 2}-\frac{11}{2} \approx \frac{1}{2}
$$

for an initial value $\Delta d^{0} \sim-0.2$ which increases to -0.4 by chiral fluctuations. The corresponding up quark distribution with chiral fluctuations is close to the dot-dashed line in Fig.1, where the exponent $\alpha=1 / 2$ and $\Delta u^{0}=4 / 3$ are chosen to simulate the NQM. It is straightforward to express the coefficients C, C' in Eq. 50 for that case in terms of A and $\alpha$ from the two constraints of Eq. 49. The formulas for the coefficients in Eq. 50 are given in the Appendix and their numerical values are displayed in Table 2. Upon imposing the observed values for $g_{A}^{(3)}, g_{A}^{(8)}$, or equivalently $\Delta u^{0}, \Delta d^{0}$ as well, the relation $\Delta u^{0}-5 \Delta d^{0}=3$ is obtained, which is satisfied by the NQM values $\Delta u^{0}=4 / 3, \Delta d^{0}=-1 / 3$. Thus, for fixed values of the exponent $\alpha$ and $\Delta u^{0}$, which are not adjusted to any observable, the chiral strength is adjusted to fit $g_{A}^{(3)}$ with chiral fluctuations, yielding the results in Figs.1,2. The case with a smaller $\Delta u^{0}=1.1$ is supposed to simulate a relativistic quark model. The price one pays for selecting a positive $x$-power is that the resulting quark distributions shown as dot-dashed line in Fig. 1 for exponent $\alpha=1 / 2$ and solid lines in Fig.2 become unrealistically small for $x \rightarrow 0$, which is a typical quark model feature. Clearly, these quark distributions have a lower peak than those of the NQM and LCQM, and it is shifted to lower $x$ values, features that make them look like evolved from quark models in Fig.1 at $\Lambda_{Q C D}$ to a higher scale. The comparison of the dotted curve in Fig. 2 which is the associated valence $u^{0}(x)$ quark distribution without chiral fluctuations with the upper solid line shows the typical size of chiral fluctuations; they shift the peak to lower $x$ but increase its magnitude for the u quark, while it is lowered for the d quark.

The integrated spin observables obtained with chiral fluctuations are given in Table 1 for the cases shown in Figs.1,2,3. Note that for the quark model simulations with exponent $\alpha=1 / 2$, despite fitting only one parameter, the chiral strength $\alpha_{\chi}$, to the axial charge of the nucleon, most of the other spin observables are not too far off. In Fig.3, though, the exponent $\alpha=0.1$ is deliberately chosen small to get closer to the BBS result for $u(x)$. For the smaller exponent $\alpha=0.1$, the total quark momentum constraint $u_{1}^{0}+d_{1}^{0}=0.52$ is imposed instead of the quark number constraints of Eq. 49, which also helps getting (the dotted line in Fig.3) closer to the BBS (dot-dashed) result, except at small $x$. For this case, nearly all integrated spin fractions in Table 1 are already in good agreement with the data, a success for which chiral fluctuations are essential in conjunction with current quark masses. Clearly, all these quark distributions become unrealistically small for $x \leq 0.1$.

When the light up and down quarks are taken to be constituent with $m_{q}=m_{N} / 3=313$ $\mathrm{MeV}$, and the strange quark with $m_{s}=m_{\Sigma}-m_{N}+m_{q}=567 \mathrm{MeV}$, then most numerical results become worse; the chiral strength has to be increased, while the proton spin $\Delta \Sigma$ moves up to about 0.5 , confirming the results of ref. [16] 
Since the recent HERA data 21] clearly show the rise of the unpolarized $F_{2}^{p}(x)$ at small $x$, which the quark model-type distributions in Figs.1,2,3 fail to reproduce, in the next step we incorporate Regge behavior imposing a negative common (isoscalar) power $x^{-\alpha_{R}}$ on the valence distributions. Just like BBS, we find that more non-leading terms must be introduced to make the proton spin fractions $\Delta q$ and the Gottfried sum rule (GSR) convergent. The minimal Regge-type parametrization is given by

$$
\begin{gathered}
u_{\uparrow}^{0}(x)=x^{-\alpha_{R}}[A+B(1-x)](1-x)^{3}, \quad u_{\downarrow}^{0}(x)=x^{-\alpha_{R}}[C+D(1-x)](1-x)^{5}, \\
d_{\uparrow}^{0}(x)=x^{-\alpha_{R}}\left[\frac{A}{5}+B^{\prime}(1-x)\right](1-x)^{3}, \quad d_{\downarrow}^{0}(x)=x^{-\alpha_{R}}\left[C^{\prime}+D^{\prime}(1-x)\right](1-x)^{5} .
\end{gathered}
$$

For $\Delta u^{0}(x), \Delta d^{0}(x)$ to be finite for $x \rightarrow 0$, the constraints

$$
A+B=C+D, \quad \frac{A}{5}+B^{\prime}=C^{\prime}+D^{\prime}
$$

are required. For the Gottfried sum rule to be convergent, or isospin symmetry for $x \rightarrow 0$, requires

$$
A+B+C+D=\frac{A}{5}+B^{\prime}+C^{\prime}+D^{\prime}
$$

Moreover, we impose fixed values for $\Delta u^{0}, \Delta d^{0}$ and the GSR, or equivalently $u_{0}^{0}-d_{0}^{0}$, and the total quark momentum $u_{1}^{0}+d_{1}^{0}$. Again, it is straightforward but tedious to determine the coefficients in Eq. 53 from these conditions; they are given analytically in the Appendix and numerically in Table 2.

Chiral fluctuations will not change the Regge exponent in the dressed quark distributions because of the asymptotic behavior of convolutions, e.g. from $q^{0}(x) \sim x^{-\alpha_{R}}$ we obtain

$$
P^{ \pm} \otimes q^{0} \sim x^{-\alpha_{R}} \int_{0}^{1} d t P^{ \pm} t^{-1-\alpha_{R}}, \quad x \rightarrow 0
$$

where the moments of the splitting functions remain finite because the Gaussian in Eq. 14 goes to zero faster than any power at the endpoints.

We adopt the Regge exponent $\alpha_{R}=1.12$ of BBS. Then we adjust the chiral strength to the spin $\Delta \Sigma$ of the proton roughly, vary $\Delta u^{0}-\Delta d^{0}, \Delta u^{0}+\Delta d^{0}$ (which changes $\Delta \Sigma$ a bit) to match the observed values for $g_{A}^{3}, g_{A}^{8}$ with chiral fluctuations, impose $u_{0}^{0}-d_{0}^{0}$ to match the GSR and $u_{1}^{0}+d_{1}^{0} \approx 1 / 2$ of the total quark momentum. The results are displayed as solid lines in Figs.3-8. The d quark distribution in Fig.4 deviates more from BBS, but the s quark distribution in Fig.5 (solid line) predicted by chiral fluctuations is again close to the BBS (dot-dashed) curve, whereas the quark model case with exponent $\alpha=0.1$ is much too small at small $x$. The spin dependent $\Delta q$ distributions in Figs.6-8 show a similar pattern. The polarized proton structure function shown in Fig.9 is crudely evolved by a multiplicative $1-\alpha_{s} / \pi \sim 0.92$ to the data at $-q^{2}=10 \mathrm{GeV}^{2}$ as in [14]. The ratio of unpolarized $F_{2}^{n} / F_{2}^{p} \rightarrow 3 / 7$ as $x \rightarrow 1$ with and without chiral fluctuations. Finally, the antiquark ratio $\bar{u} / \bar{d}$ in Fig.10 is not even approximately constant. 


\section{CONCLUSIONS}

A comparison of the chiral field theory of quark structure functions with previous chiral models [5, 10 12,6] for the spin fractions $\Delta q$ shows unambiguously that the remarkable success of these models is due to parametrizations that correspond to neglecting helicity non-flip chiral fluctuations in the (integrated) polarized splitting functions which are sizable for constituent quarks. Thus, standard chiral dynamics can explain the spin observables of the proton only for the case where the dynamical quark masses are already small. Furthermore, the present analysis sheds light on the failure of standard chiral dynamics at the

scale $\Lambda_{Q C D}$ involving constituent quarks [16] whose larger masses significantly reduce the negative chiral contributions to the spin fractions. When quark distributions are constructed according to the quark counting rules of pQCD in conjunction with constraints from the $\mathrm{SU}(6)$ spin-flavor wave function of the proton, the GSR and total quark momentum fraction, then chiral dynamics generates sea quark distributions of correct magnitude and shapes and predicts reasonable strange quark distributions in particular so that the spin fractions of the proton also agree with the data.

While chiral dynamics with the (ad-hoc) pure spinflip prescription appears to be successful in understanding the proton spin fractions, its justification in the context of chiral quark models may be provided by instanton dynamics of zero-mode quarks. In fact, 't Hooft's 23] interaction maintains pure left- to right-handed quark transitions, while light quarks pick up a dynamical mass upon propagating in the instanton background field. [24] However, axialvector coupling of constituent quarks with Goldstone bosons in standard chiral field theory is inconsistent with the proton spin fraction data.

\section{APPENDIX}

The coefficients of the valence quark distributions for the cases of Fig.1 (dot-dashed line) and Fig.2 (upper solid and dotted lines) with $\alpha=1 / 2$ are given by

$$
\begin{gathered}
A=\frac{2+\Delta u^{0}}{12} \Pi_{j=1}^{4}(j+\alpha), \quad C=\frac{2-\Delta u^{0}}{240} \Pi_{j=1}^{6}(j+\alpha), \\
C^{\prime}=\frac{8-\Delta u^{0}}{1200} \Pi_{j=1}^{6}(j+\alpha),
\end{gathered}
$$

those for Fig.3 (dotted line) with $\alpha=0.1$ and total quark momentum constraint are

$$
\begin{gathered}
A=\frac{10}{147}\left[\left(u_{1}^{0}+d_{1}^{0}\right)(5+\alpha)+\left(\Delta u^{0}+\Delta d^{0}\right)(1+\alpha)\right] \Pi_{j=2}^{4}(j+\alpha), \\
C=\frac{A}{20}(6+\alpha)(5+\alpha)-\frac{\Delta u^{0}}{120} \Pi_{j=1}^{6}(j+\alpha), \\
C^{\prime}=\frac{A}{100}(6+\alpha)(5+\alpha)-\frac{\Delta d^{0}}{120} \Pi_{j=1}^{6}(j+\alpha),
\end{gathered}
$$


while those of the Regge (solid lines in Figs.3-8) and BBS (dot-dashed lines in Figs.2-8) are given by

$$
\begin{gathered}
A=\frac{5}{48}\left(\Delta u^{0}-\Delta d^{0}+u_{0}^{0}-d_{0}^{0}\right) \Pi_{j=2}^{5}\left(j-\alpha_{R}\right), \\
\frac{-40 B}{A}\left[\left(12-\alpha_{R}\right)\left(2-\alpha_{R}\right)+\left(7-\alpha_{R}\right)\left(8-\alpha_{R}\right)+30\right]=\left[6\left(6-\alpha_{R}\right)\left(7-\alpha_{R}\right)\left(8-\alpha_{R}\right)\right. \\
\left.+16\left(7-\alpha_{R}\right)\left(8-\alpha_{R}\right)+1200+\left(2-\alpha_{R}\right)\left(732-118 \alpha_{R}+6 \alpha_{R}^{2}\right)\right] \\
+\frac{1}{6} \Pi_{j=3}^{7}\left(j-\alpha_{R}\right)\left[\left(u_{1}^{0}+d_{1}^{0}\right)\left(8-\alpha_{R}\right)+\left(\Delta u^{0}+\Delta d^{0}\right)\left(2-\alpha_{R}\right)\right], \\
40 C=-\frac{\Delta u^{0}}{3} \Pi_{j=2}^{7}\left(j-\alpha_{R}\right)+8 B\left(12-\alpha_{R}\right) \\
+\frac{A}{5}\left[4\left(7-\alpha_{R}\right)\left(6-\alpha_{R}\right)+732-118 \alpha_{R}+6 \alpha_{R}^{2}\right], \\
40 C^{\prime}=-\frac{\Delta d^{0}}{3} \Pi_{j=2}^{7}\left(j-\alpha_{R}\right)+8 B\left(12-\alpha_{R}\right) \\
+\frac{A}{5}\left[732-118 \alpha_{R}+6 \alpha_{R}^{2}-4\left(7-\alpha_{R}\right)\left(6-\alpha_{R}\right)\right] . \\
B^{\prime}=\frac{4}{5} A+B, \quad D^{\prime}=A+B-C^{\prime} .
\end{gathered}
$$


Table 1 Quark Spin Fractions of the Proton for Figs.1-8

\begin{tabular}{|c|c|c|c|c|c|}
\hline & $\begin{array}{c}\text { Data E143 [8] } \\
\text { at } 3 \mathrm{GeV}^{2} \\
\text { Data } \mathrm{SMC}[9] \\
\text { at } 5 \mathrm{GeV}^{2}\end{array}$ & $\begin{array}{c}\alpha_{\chi}=0.39 \\
\alpha=\frac{1}{2} \\
\Delta u^{0}=\frac{4}{3} \\
\text { Figs. } 1.2\end{array}$ & $\begin{array}{c}\alpha_{\chi}=0.3 \\
\alpha=\frac{1}{2} \\
\Delta u^{0}=1.1 \\
\text { Figs. } 2\end{array}$ & $\begin{array}{c}\alpha_{\chi}=0.42 \\
\alpha=0.1 \\
\text { Figs.3-8 }\end{array}$ & $\begin{array}{c}\alpha_{\chi}=0.4 \\
\alpha_{R}=1.12 \\
\text { Fig.3-8 }\end{array}$ \\
\hline$\Delta u$ & $\begin{array}{l}0.84 \pm 0.05 \\
0.82 \pm 0.02\end{array}$ & 0.82 & 0.86 & 0.86 & 0.82 \\
\hline$\Delta d$ & $\begin{array}{l}-0.43 \pm 0.05 \\
-0.43 \pm 0.02\end{array}$ & -0.44 & -0.40 & -0.40 & -0.44 \\
\hline$\Delta s$ & $\begin{array}{l}-0.08 \pm 0.05 \\
-0.10 \pm 0.02\end{array}$ & -0.04 & -0.02 & -0.07 & -0.11 \\
\hline$\Delta \Sigma$ & $\begin{array}{l}0.30 \pm 0.06 \\
0.29 \pm 0.06\end{array}$ & 0.344 & 0.44 & 0.39 & 0.27 \\
\hline$\Delta_{3} / \Delta_{8}$ & $2.09 \pm 0.13$ & 2.76 & 2.06 & 2.08 & 2.11 \\
\hline$g_{A}^{(3)}$ & $1.2601 \pm 0.002522$ & 1.254 & 1.263 & 1.254 & 1.254 \\
\hline $\mathcal{F} / \mathcal{D}$ & $0.575 \pm 0.016$ & 0.52 & 0.53 & 0.59 & 0.58 \\
\hline$I_{G}$ & $0.235 \pm 0.026$ & 0.283 & 0.304 & 0.244 & 0.286 \\
\hline
\end{tabular}


Table 2 Coefficients of Valence Quark Distributions in Eqs. 50,53 for Figs.1-8

\begin{tabular}{|c|c|c|c|c|c|}
\hline & $\begin{array}{c}\text { BBS [14] } \\
\text { Figs.2-8 }\end{array}$ & $\begin{array}{c}\alpha_{\chi}=0.39 \\
\alpha=\frac{1}{2} \\
\Delta u^{0}=\frac{4}{3} \\
\text { Figs. } 1,2\end{array}$ & $\begin{array}{c}\alpha_{\chi}=0.3 \\
\alpha=\frac{1}{2} \\
\Delta u^{0}=1.1 \\
\text { Figs.2 }\end{array}$ & $\begin{array}{c}\alpha_{\chi}=0.42 \\
\alpha=0.1\end{array}$ & $\begin{array}{c}\alpha_{\chi}=0.4 \\
\alpha_{R}=1.12\end{array}$ \\
\hline Aigs.3-8 & Figs.3-8 \\
\hline B & 3.784 & 16.406 & 15.258 & 8.091 & 5.565 \\
B' & -3.672 & - & - & - & -5.551 \\
C & -0.645 & - & - & - & -1.099 \\
C' & 2.004 & 5.866 & 7.918 & -0.354 & -1.047 \\
D & 3.230 & 11.730 & 12.141 & 2.974 & 0.920 \\
D' & -1.892 & - & - & - & 1.061 \\
\hline
\end{tabular}




\section{FIGURES}

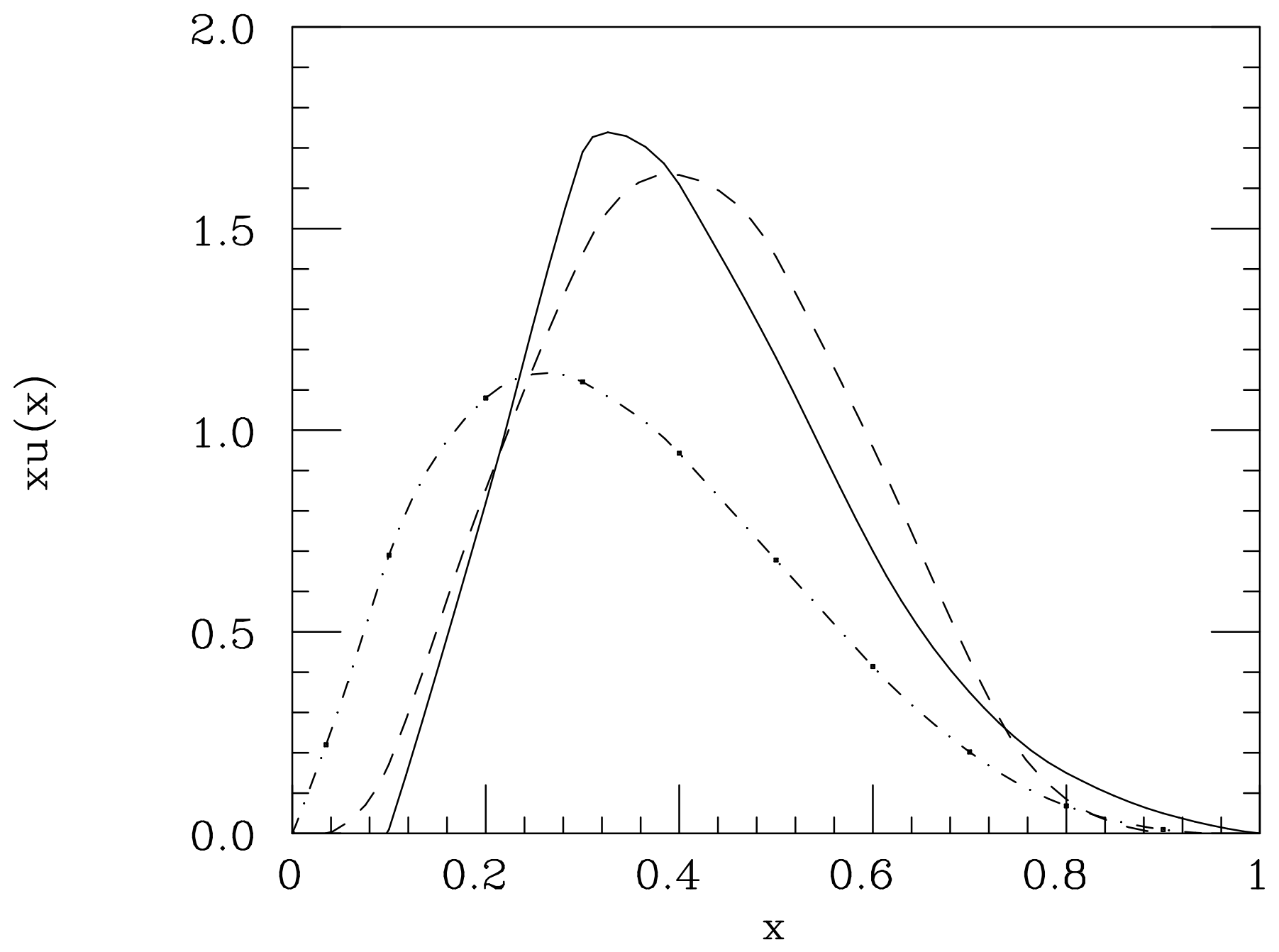

FIG. 1. Quark distribution $x u(x)$ of NQM [18] (solid), LCQM [11] (dashed), both at $\Lambda_{Q C D}$, case of 3 rd column of Table 1 (dot-dashed) with chiral fluctuations at $\Lambda_{\chi}$. 


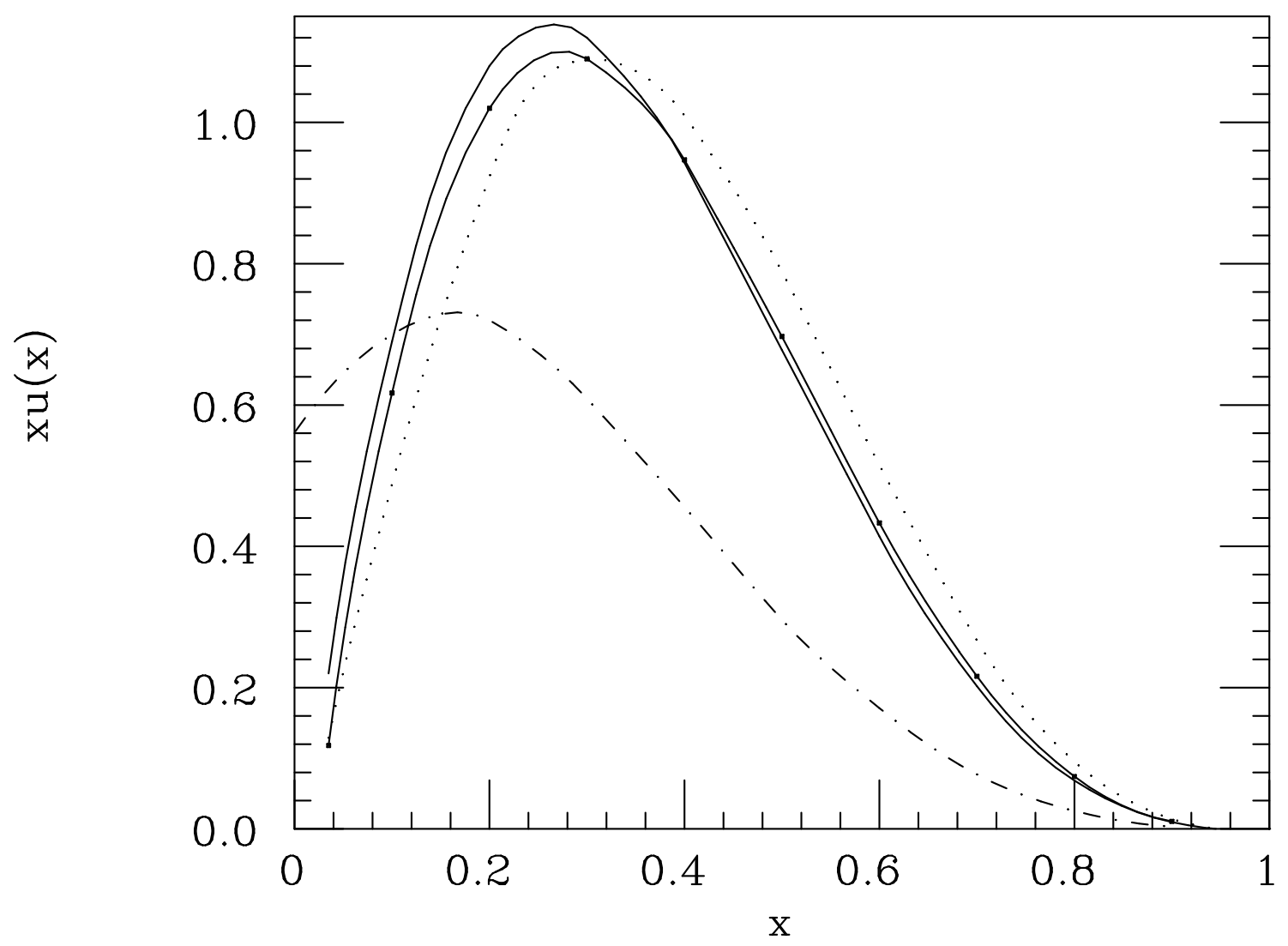

FIG. 2. Quark distribution $x u(x)$ of 3rd column of Table 1 (top solid) with corresponding valence $x u^{0}(x)$ (dotted) without chiral fluctuations, case of 4th column of Table 1 (lower solid) compared with BBS [12] (dot-dashed). 


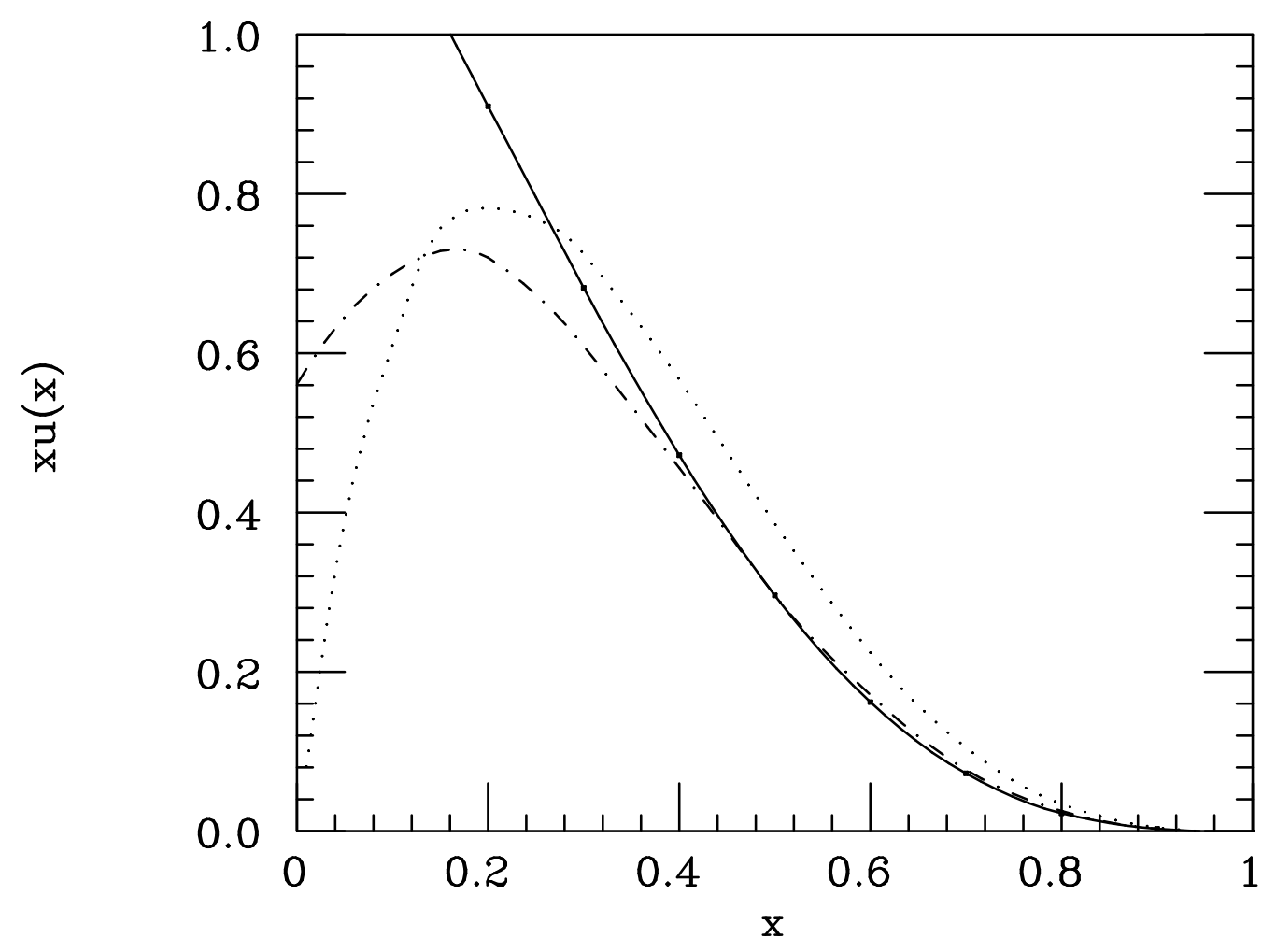

FIG. 3. Quark distribution $x u(x)$ of 5th column of Table 1 (dotted), Regge case of last column of Table 1 (solid) compared with BBS [12] (dot-dashed). 


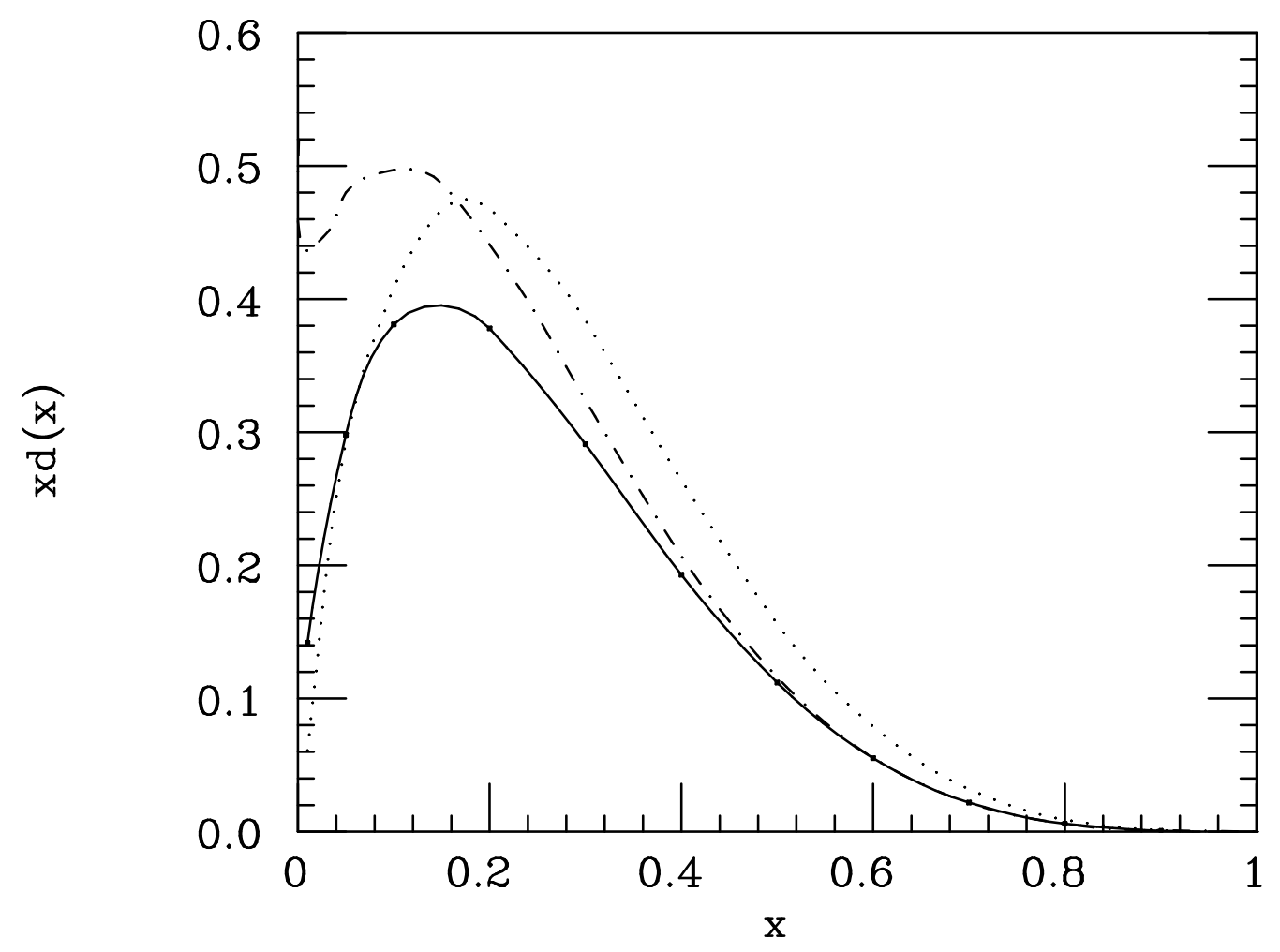

FIG. 4. Same as Fig.3 for down quark $x d(x)$. 


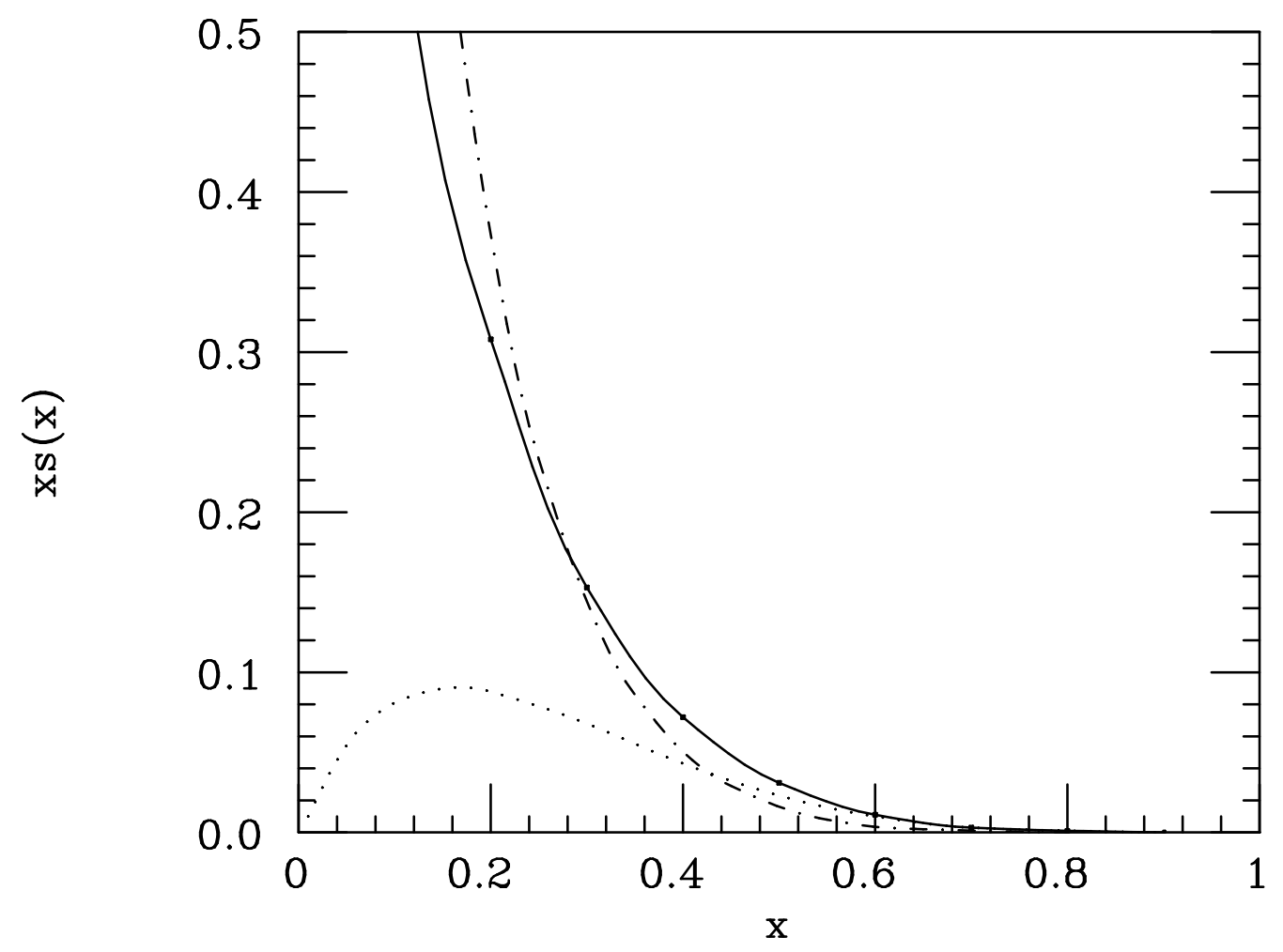

FIG. 5. Same as Fig.3 for strange quark $x s(x)$. 


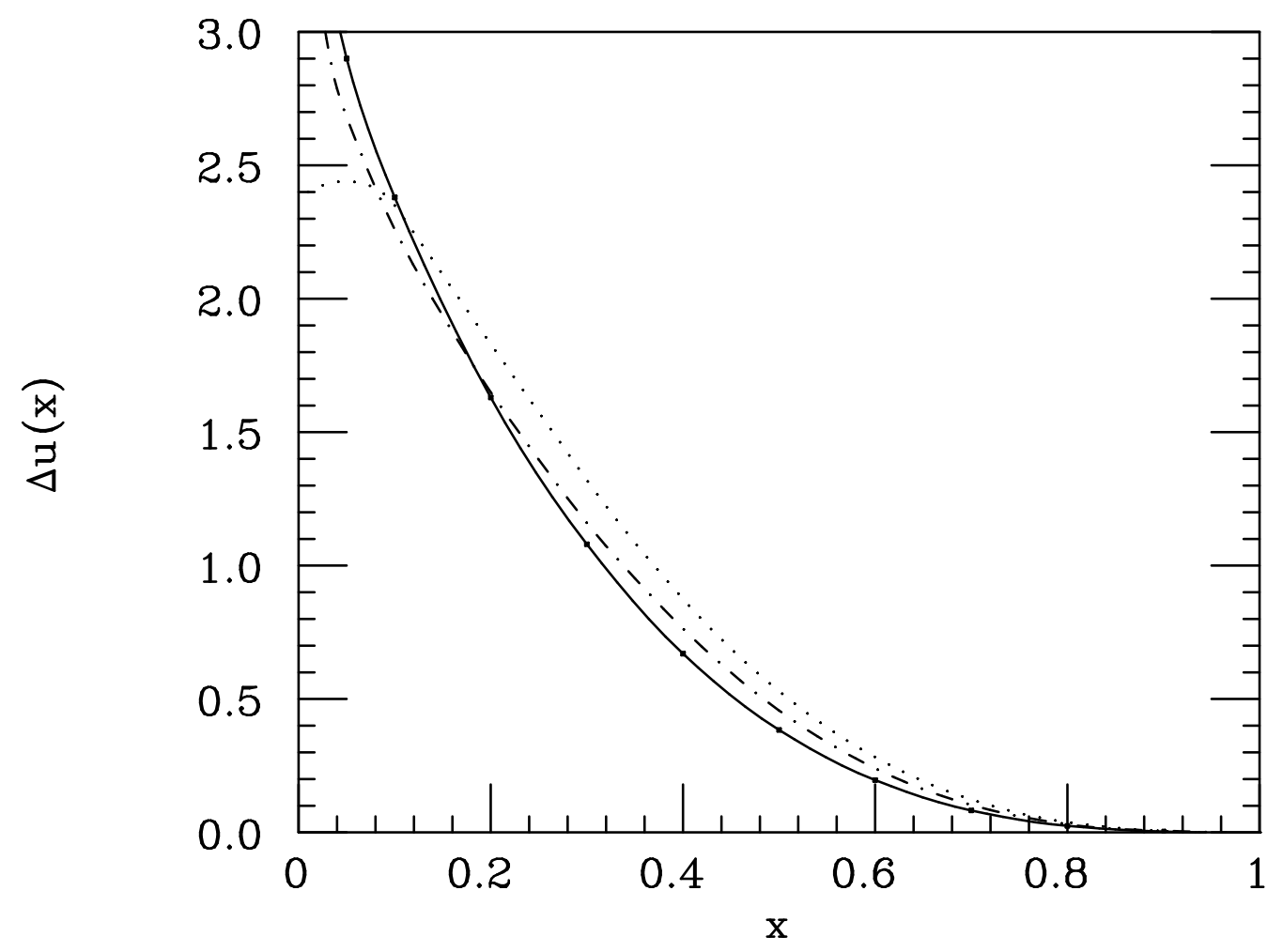

FIG. 6. Same as Fig.3 for polarized $\Delta u(x)$. 


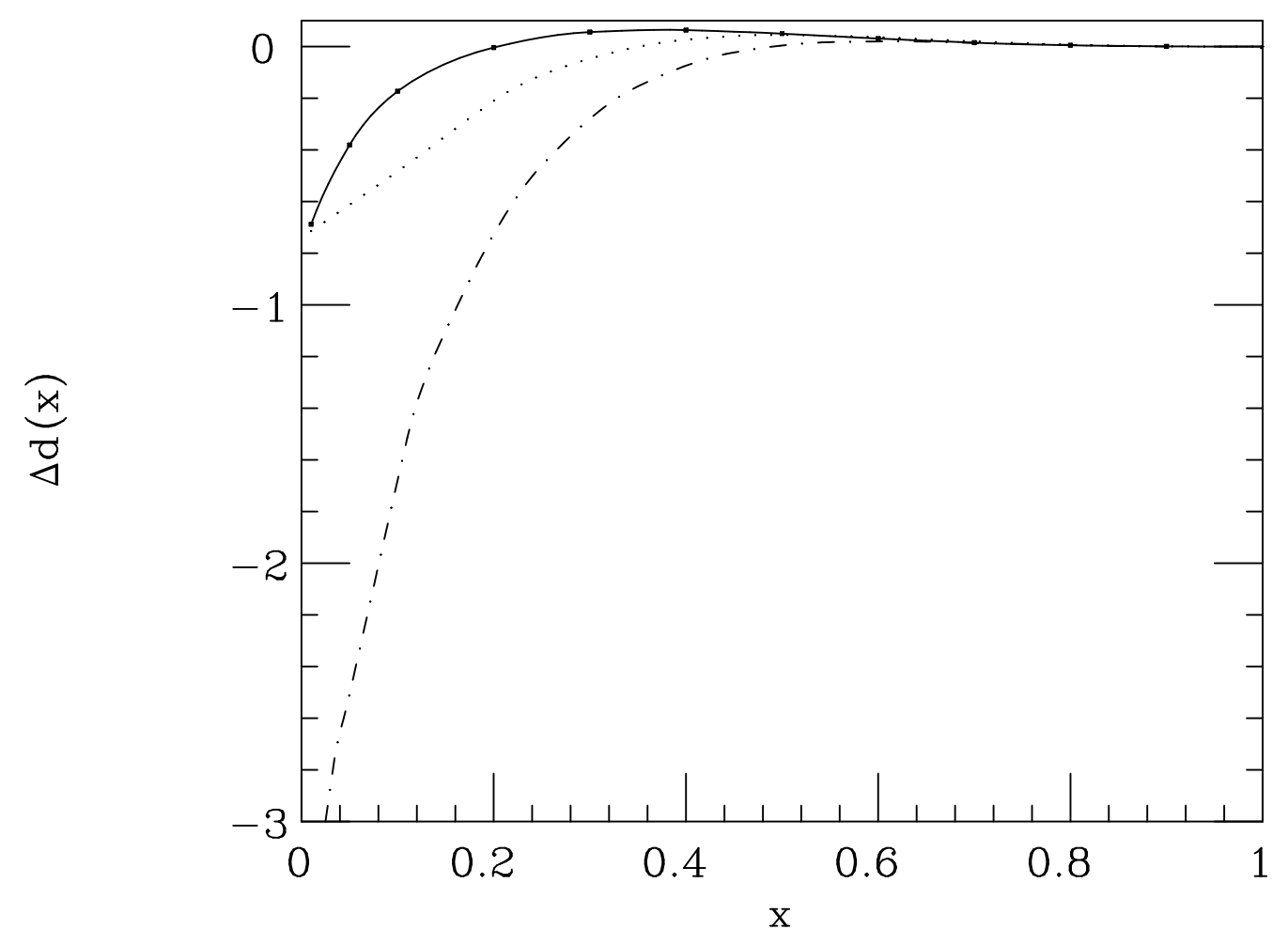

FIG. 7. Same as Fig.3 for polarized $\Delta d(x)$. 


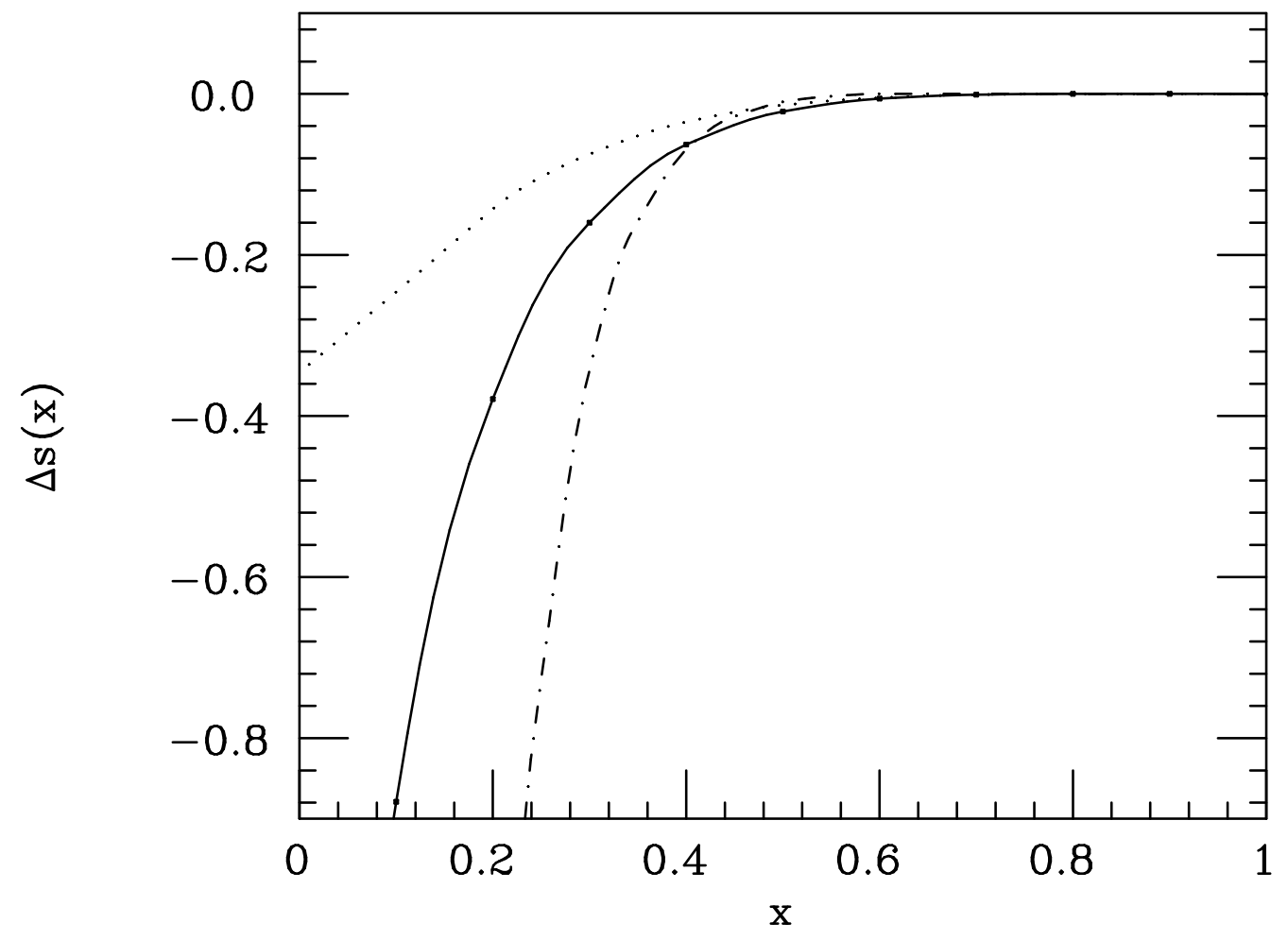

FIG. 8. Same as Fig.3 for polarized $\Delta s(x)$. 


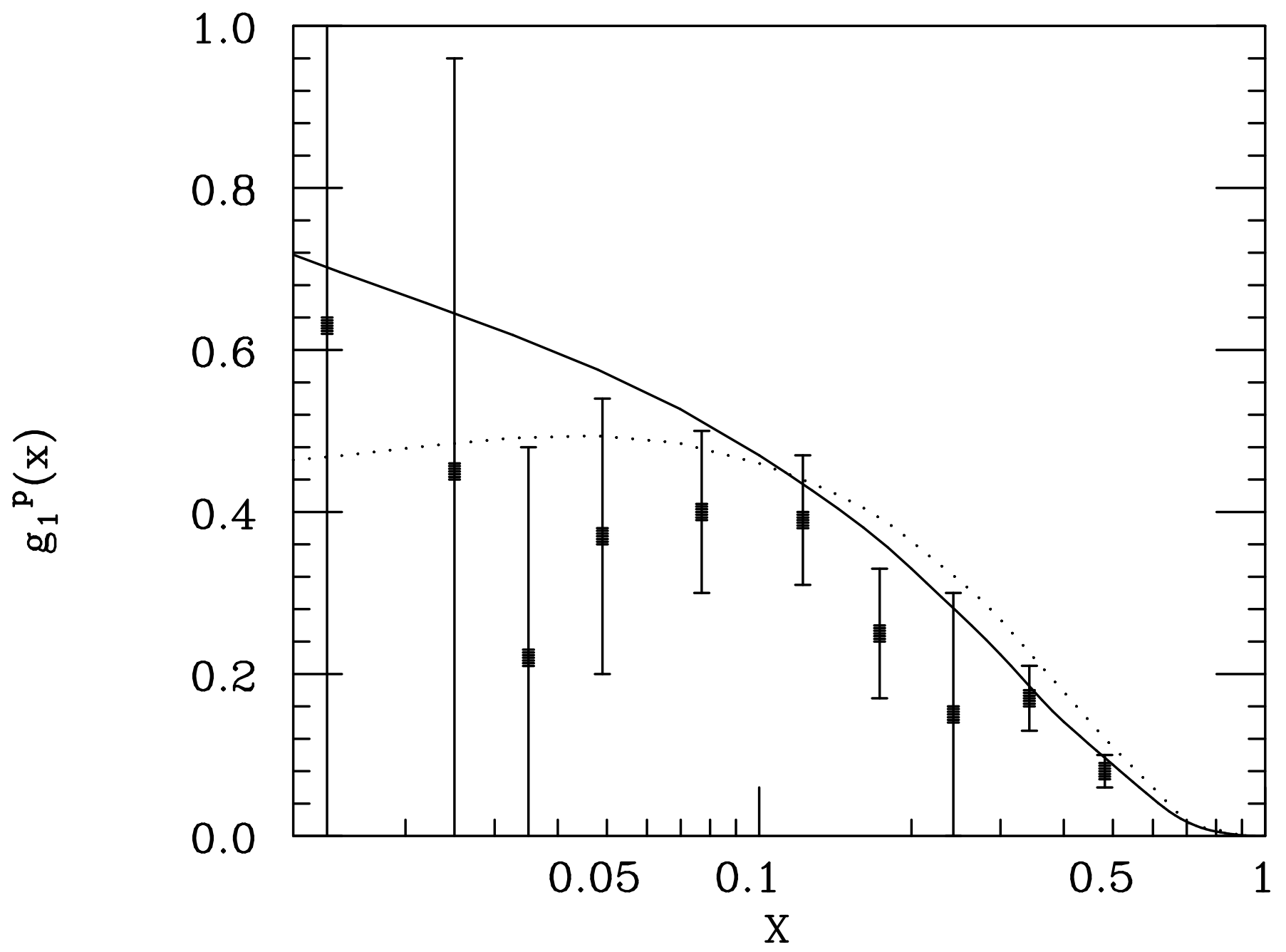

FIG. 9. Polarized structure function $g_{1}^{p}(x)$ of the proton; notation as in Fig.3. Data are from SMC, hep-ex/9702005. 


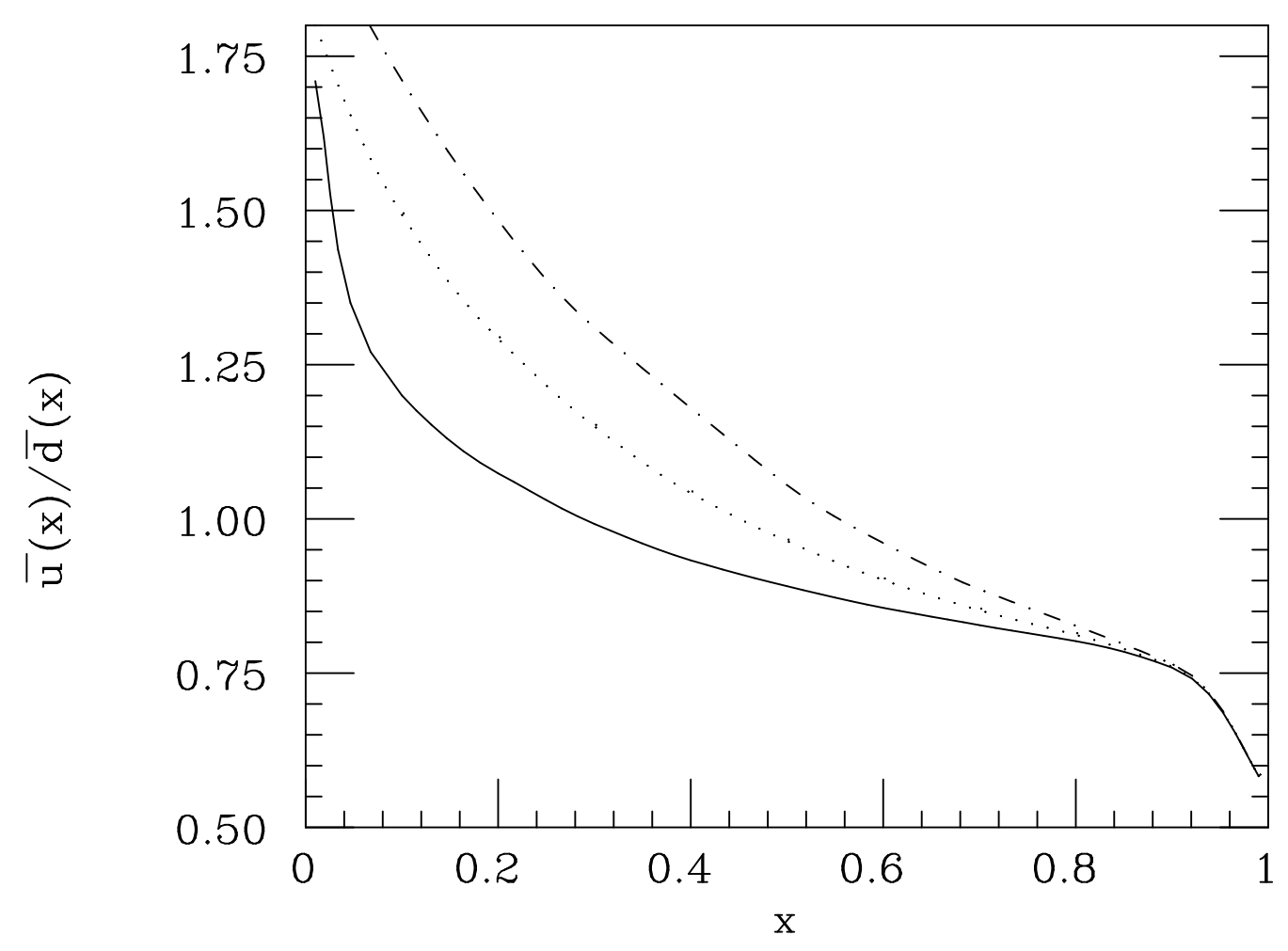

FIG. 10. Same as Fig.3 for the antiquark ratio $\bar{u}(x) / \bar{d}(x)$ 


\section{REFERENCES}

[1] T. Hatsuda and T. Kunihiro, Phys. Rep. 247, 221 (1994).

[2] G. 't Hooft, Nucl. Phys. B190, 455 (1981), ibid. B153, 141 (1979); S. Mandelstam, Phys. Rev. D19, 2391 (1979); Y. Nambu, ibid. D10, 4262 (1974); T. Suzuki, Progr. Theor. Phys. 80, 929 (1988).

[3] S. Umisedo, H. Suganuma and H. Toki, hep-ph/9710231.

[4] H. Leutwyler, Ann. Phys.(N.Y.) 235, 165 (1994).

[5] E. J. Eichten, I. Hinchcliffe and C. Quigg, Phys. Rev. D45, 2269 (1992).

[6] H. J. Weber and K. Bodoor, Int. J. Mod. Phys. E6, 693 (1997), hep-ph/9707270.

[7] K. Gottfried, Phys. Rev. Lett. 18, 1174 (1967).

[8] P. L. Anthony et al. (E142), Phys. Rev. Lett. 71, 959 (1993), Phys. Rev. D54, 6620 (1996); K. Abe et al. (E143), Phys. Rev. Lett. 74, 346 (1995); ibid. 75, 25 (1995); K. Abe et al. (E154), Phys. Rev. Lett. 79, 26 (1997).

[9] B. Adeva et al. (SMC), Phys. Lett. B302, 553 (1993); ibid. B320, 400 (1994); ibid. 369, 93 (1996); D. Adams et al., Phys. Lett. B329, 399 (1994); ibid. B336, 125 (1994); D. Adams et al., Phys. Lett. B357, 248 (1995), ibid. B396, 338 (1997), Phys. Rev. D56, 5330 (1997).

[10] H. J. Weber, X. Song, and M. Kirchbach, Mod. Phys. Lett. A12, 729 (1997), hep$\mathrm{ph} / 9701266$.

[11] X. Song, J. S. McCarthy, and H. J. Weber, Phys. Rev. D55, 2624 (1997), and refs. therein.

[12] T. P. Cheng and L.-F. Li, Phys. Rev. Lett. 74, 2872 (1995).

[13] H. J. Weber, Phys. Rev. D 49, 3160 (1994).

[14] S. J. Brodsky, M. Burkhardt, I. Schmidt, Nucl. Phys. B44, 197 (1995).

[15] E. Leader, A. V. Sidorov and D. B. Stamenov, hep-ph/9708335.

[16] K. Suzuki and W. Weise, Nucl. Phys. A634, 141 (1998), hep-ph/9711368, and refs. therein.

[17] W. Melnitchouk and M. Malheiro, Phys. Rev. C55, 431 (1997), hep-ph/9610331 and refs. therein.

[18] S. J. Brodsky and B.-Q. Ma, Phys. Lett. B 381, 317 (1996).

[19] M. Traini, L. Conci and U. Moschella, Nucl. Phys. A544, 731 (1992).

[20] M. Glück, E. Reya and A. Vogt, Z. Phys. C67, 433 (1995).

[21] J. Breitweg et al. (ZEUS), Phys. Lett. B407, 432 (1997); M. Derrick et al., Z. Phys. C69, 607 (1996), ibid. C72, 394 (1996); S. Aid et al. (H1), Nucl. Phys. B470, 3 (1996), ibid. B497, 3 (1997).

[22] Particle Data Group, R. M. Barnett et al., Phys. Rev. D54, 1 (1996).

[23] G. 't Hooft, Phys. Rev. D14, 3432 (1976).

[24] A. E. Dorokhov, N. I. Kochelev and Yu. A. Zubov, Mod. Phys. A8, 603 (1993), and refs. therein. 